\title{
Application of the Complex Variable Semi-analytical Method for Improved Displacement Sensitivity Evaluation in Geometrically Nonlinear Truss Problems
}

Abstract

The application of gradient-based methods to structural optimization problems usually requires the determination of displacement sensitivities with respect to design variables. In this regard, it is important to have at hand comprehensive methods for sensitivity analysis, which show stability, efficiency and accuracy. Particularly, application of the semi-analytical method for linear and nonlinear problems is generally a good trade-off between formulation simplicity and accuracy. In spite of that, semi-analytical methods are known to behave pathologically for shape design variables when the structure is subjected to rigid rotations. A large number of solutions for this problem have been presented in last years, although the formulation involved is generally not trivial, especially in the nonlinear case. A recent method, which adopts the semianalytical approach and uses complex variables has rendered very promising results for all the aforementioned aspects: stability, efficiency and accuracy. Additionally, it is simple to codify. The present contribution is concerned with the application of this sensitivity analysis method to geometrically nonlinear truss problems. To this end, a finite element formulation is presented and displacement sensitivities are evaluated with respect to material and shape design variables. The results are compared to those obtained using the semi-analytical method with real variables and to global finite differences. An example demonstrates the potentiality of this new approach.

\section{Keywords}

Structural optimization, Sensitivity analysis, Finite element method, Nonlinear analyses.

\section{Geovane Augusto Haveroth ${ }^{\text {a }}$ \\ Joânesson Stahlschmidt ${ }^{b}$ \\ Pablo Andrés Muñoz-Rojas ${ }^{c}$}

Departamento de Eng. Mecânica, Universidade do Estado de Santa Catarina, UDESC, Campus Univ. Avelino Marcante, CEP 90226-100, Joinville, SC, Brazil

a geovaneah@gmail.com

b joanesson@ibest.com.br

c pablo@joinville.udesc.br

http://dx.doi.org/10.1590/1679-78251911

Received 11.02.2015

Accepted 01.04.2015

Available online 19.04.2015 


\section{INTRODUCTION}

Sensitivity analysis is concerned with the evaluation of gradients of a structural response with respect to design variables. The response of interest may be the field of displacements, stresses or strains, a natural frequency, a bucking load, and so on. Typical design variables are shape or material parameters, which can be modified in the design stage, such that their change affects the overall behavior of the structure (Kleiber et al., 1997). Many methods have been developed to accomplish the sensitivity evaluation task, and for linear problems, a high level of maturity has already been achieved. This is not exactly the situation in problems that involve some kind of nonlinearity, and an important research field is still open in this case (Choi and Kim, 2005).

Methods for sensitivity analysis can be classified into three different groups: analytical methods, semi-analytical methods and overall finite differences. It is important to remark that the use of the traditional semi-analytical method for the evaluation of sensitivities with respect to shape design variables can provide unacceptable errors when the structure suffers large rigid body rotations, as reported in a large number of publications. In the case of linear problems, this pathology has been the subject of studies by Barthelemy et al. (1988), Pedersen et al. (1989), Cheng and Olhoff (1991), Mlejnek (1992), Olhoff et al. (1993), Keulen et al. (2005), Bletzinger et al. (2007), and many more. De Boer and Keulen (2000) and Parente and Vaz (2001), among others have proposed some remedies for the drawback in the case of nonlinear problems.

Recently, a modification of the semi-analytical method introducing the use of complex variables has been successfully applied to solve the mentioned pitfall (Jin, 2008; Jin et al., 2009; Jin et al., 2010). Their study dealt with linear and a few nonlinear heat, beam and plate problems, and showed accuracy as well as efficiency, since only a marginal extra computational cost is required. An important feature of the method is that it allows extremely small numerical perturbations without compromising accuracy.

The present article aims to show how the semi-analytical complex variable sensitivity analysis method behaves when applied to geometrically non-linear truss problems. To this end, the corresponding nonlinear finite element formulation is developed and detailed. Here it is important to remark that nonlinear sensitivity analysis requires the use of the exact tangent stiffness matrix, as will be shown. Displacement sensitivities with respect to material and shape design variables are studied in a problem dominated by rigid rotations. Results are compared to the semi-analytical method using real variables and to overall finite differences. For conciseness, the following acronyms are used throughout the text: CVSA (Complex Variable Semi-Analytical), RVSA (Real Variable Semi-Analytical), FFD (Forward Finite Difference), CFD (Central Finite Difference) and OFD (Overall Finite Difference). In this work, the CVSA method is always based on FFD.

\section{GEOMETRICALLY NONLINEAR FINITE ELEMENT FORMULATION FOR TRUSSES}

The evaluation of displacement sensitivities in nonlinear problems is directly affected by the proper calculation of the tangent stiffness matrix, which should be obtained via exact linearization of the internal force vector. Any approximation adopted in the internal force differentiation results in inexact evaluation of gradients, as will be emphasized in Section 3.1. For now, it suffices to mention the fact to justify the attention given to the linearization procedure described in Sections 2.1 to 2.3. 
In Fig. 1, a typical 2 noded truss element is displayed, and 2 associated systems of reference are defined: a still, global system of reference $X Y$ and a corotational local system of reference $x_{l} y_{l}$. A parametric coordinate $\xi$ is also defined along the length of the bar.

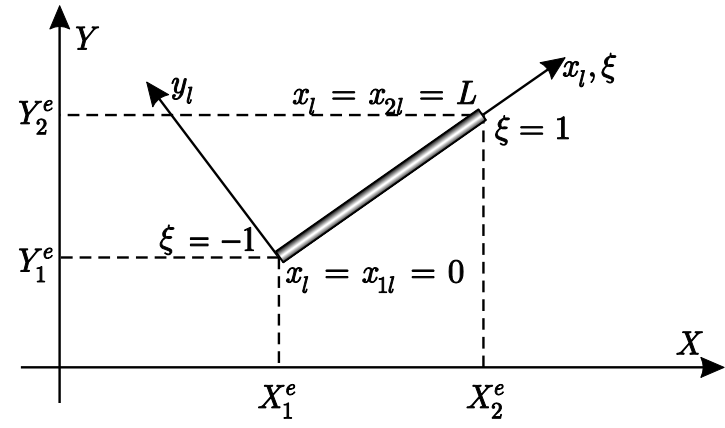

Figure 1: Bar finite element.

The local coordinates and displacements of any given point in the undeformed element are obtained applying a linear interpolation of the nodal values, i.e.,

$$
X_{l}=\boldsymbol{N}(\xi) \boldsymbol{X}_{l} \quad \text { and } \quad u_{l}=\boldsymbol{N}(\xi) \boldsymbol{u}_{l},
$$

so that at any time, the updated rotated bar geometry can be expressed by

$$
x_{l}=X_{l}+u_{l}=\boldsymbol{N}(\xi) \boldsymbol{x}_{l}
$$

where

$$
\begin{gathered}
\boldsymbol{N}(\xi)=\left[\begin{array}{llllll}
N_{1}(\xi) & 0 & 0 & N_{2}(\xi) & 0 & 0
\end{array}\right], \\
\boldsymbol{X}_{l}=\left[\begin{array}{llllll}
X_{1} & Y_{1} & Z_{1} & X_{2} & Y_{2} & Z_{2}
\end{array}\right]^{T}, \\
\boldsymbol{u}_{l}=\left[\begin{array}{llllll}
u_{x 1 l} & u_{y 1 l} & u_{z 1 l} & u_{x 2 l} & u_{y 2 l} & u_{z 2 l}
\end{array}\right]^{T}
\end{gathered}
$$

and

$$
\boldsymbol{x}_{l}=\left[\begin{array}{llllll}
x_{1 l} & y_{1 l} & z_{1 l} & x_{2 l} & y_{2 l} & z_{2 l}
\end{array}\right]^{T}
$$

with $N_{1}$ and $N_{2}$ being the linear interpolation functions

$$
N_{1}(\xi)=\frac{1-\xi}{2} \text { and } \quad N_{2}(\xi)=\frac{1+\xi}{2}
$$

Additionally, using (1) and (3), the corresponding Jacobians are expressed by

$$
J\left(X_{l}\right)=\frac{d X_{l}}{d \xi}=\frac{L_{o}}{2} \quad \text { and } \quad J\left(x_{l}\right)=\frac{d x_{l}}{d \xi}=\frac{L}{2},
$$

where $L_{o}$ and $L$ are the undeformed and deformed (current geometry) length of the bar. 
In a nonlinear FE formulation, at each $n$-th load step, equilibrium must be satisfied. Thus, minimization of the residual $\boldsymbol{R}_{n}$ (difference between external and internal forces) is sought for, that is,

$$
\boldsymbol{R}_{n}=\mu_{n} \boldsymbol{p}-\boldsymbol{f}\left(\boldsymbol{u}_{n}\right) \approx \mathbf{0},
$$

where $\boldsymbol{f}$ is the global internal force vector, $n$ stands for the load step and $\mu$ is a parameter that controls the percentage of the total global external load vector $\boldsymbol{p}$ applied at the $n$-th step. The absence of subscripts $l$ indicates that the equations are set in the global system of reference (components in $X$ and $Y$ directions).

Taking the exact linearization of (12) by means of a Taylor series expansion truncated at first order terms with origin at $\tilde{\boldsymbol{u}}_{n}^{\alpha-1}$, results in

$$
\boldsymbol{R}_{n}\left(\tilde{\boldsymbol{u}}_{n}^{\alpha}\right)=\boldsymbol{R}_{n}\left(\tilde{\boldsymbol{u}}_{n}^{\alpha-1}+\Delta \boldsymbol{u}_{n}\right) \approx \boldsymbol{R}_{n}\left(\tilde{\boldsymbol{u}}_{n}^{\alpha-1}\right)+\frac{d \boldsymbol{R}_{n}\left(\tilde{\boldsymbol{u}}_{n}^{\alpha-1}\right)}{d \tilde{\boldsymbol{u}}_{n}^{\alpha-1}} \Delta \boldsymbol{u}_{n} \approx \mathbf{0}
$$

where $\alpha$ stands for the iteration number.

Starting at a prescribed arbitrary initial point $\tilde{u}_{n}^{0} \quad \alpha=1$ ), which in general does not satisfy equilibrium, (13) must be solved iteratively until the residual falls into a prescribed tolerance range (the tilde indicates that the value is not converged). The determination of $\Delta \boldsymbol{u}_{n}$ requires the evaluation of the residual derivative with respect to $\tilde{\boldsymbol{u}}_{n}^{\alpha-1}$. This derivative is called stiffness tangent matrix and is obtained by differentiation of the external and internal force vectors. Aiming at the evaluation of (12) and (13), the expressions of the internal force vector and tangent matrix are derived in the remainder of this Section.

\subsection{Virtual Work}

One traditional manner to set up the conditions that grant equilibrium to a deformable body is the application of the Principle of Virtual Work. This principle states that the work performed by a real external force applied on a point of the body, over an imaginary (virtual) and arbitrary displacement of the point $\left(\delta W_{\text {ext }}\right)$, must equal the work performed by internal forces in equilibrium with the real force applied, over the displacement field in equilibrium with the prescribed virtual external displacement.

The expression for the virtual work resultant from a virtual displacement on a bar that is already subjected to a force $P$ is easily developed, as seen in Fig. 2.

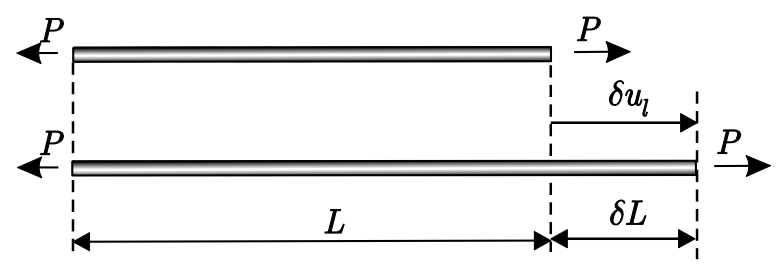

Figure 2: External virtual work. 
While the external work ( $\left.\delta W_{\text {ext }}\right)$ acting on the bar in Fig. 2 is given by

$$
\delta W_{e x t}=P \delta u_{l}
$$

the internal virtual work $\delta W_{i}$ can be expressed using different stress and strain definitions, provided that their joint use leads to the same virtual work given by $\delta W_{e x t}$. Stress and strain measures that satisfy this condition are said to be energetically or work conjugate. The rotated engineering stress and engineering strain measures are defined as ${ }^{1}$

$$
\sigma_{E}=\frac{F}{A_{0}} \quad \text { and } \quad \varepsilon_{E}=\frac{d x_{l}-d X_{l}}{d X_{l}} .
$$

Using (10) and (11), it turns out that

$$
\varepsilon_{E}=\frac{J\left(x_{l}\right)-J\left(X_{l}\right)}{J\left(X_{l}\right)}=\frac{L-L_{o}}{L_{o}}=\frac{u_{l}}{L_{o}},
$$

where a linear displacement profile is assumed. Therefore, the virtual strain resulting from a virtual displacement is

$$
\delta \varepsilon_{E}=\frac{d \varepsilon_{E}}{d u_{l}} \delta u_{l}=\frac{1}{L_{o}} \delta u_{l}
$$

and the following identities hold

$$
\delta W_{e x t}=P \delta u_{l}=(\sigma A) \delta u_{l}=\frac{P}{A_{o}} A_{o} \delta u_{l}=\left(\sigma_{E} A_{o}\right) \delta u_{l}=\sigma_{E} \delta \varepsilon_{E} A_{o} L_{o}=\delta W_{i},
$$

where $\sigma$ is the rotated Cauchy stress measure, and $A_{o}$ and $A$ are the undeformed and deformed areas of the cross section, respectively.

In (19) it is implicit that the stress value is the same along the length of the bar. Generalization leads to

$$
\delta W_{i}=\int_{V_{o}} \sigma_{E} \delta \varepsilon_{E} d V_{o}
$$

and the rotated engineering stress and strain pair is shown to be work conjugate. In this derivation, a given entity is said to be rotated if it is referred to the corotational system of reference.

\footnotetext{
${ }^{1}$ In bar structural elements, out of all the components of the stress tensor, only $\sigma_{11}=\sigma_{x l}$ is not null and produces work. Thus, in this text, the scalar stress measures cited are always the component $\sigma_{x l}$ of the respective stress tensors. The same applies for the strain tensors.
}

Latin American Journal of Solids and Structures 12 (2015) 980-1005 


\subsection{Internal Force Vector}

Generalizing the derivation presented in Section 2.1 to a truss structure, the use of (1) and (2) leads to the discretized version of the principle of virtual work

$$
\delta W_{e x t}=\underbrace{\sum_{e=1}^{n e l} \delta \boldsymbol{u}_{l}^{T} \boldsymbol{f}_{l}}_{\delta W_{i}}=\underbrace{\sum_{e=1}^{n e l} \int_{V_{o}} \delta \varepsilon_{E} \sigma_{E} d V_{o}}_{\delta W_{i}}=\begin{gathered}
\delta \boldsymbol{u}^{T} \boldsymbol{p}, \\
\delta W_{e x t}
\end{gathered},
$$

where $e$ stands for a given element, nel is the total number of elements and $\delta W_{\text {ext }}$ now stands for the virtual work caused by the virtual displacement $\delta \boldsymbol{u}$. The $\boldsymbol{f}_{l}$ vector contains the components of the element nodal internal force with respect to the local system of reference and $\boldsymbol{p}$ is a vector containing the external applied loads with components referenced to the global system of reference. In the particular situation shown in Fig. 2 and equation (14), $\boldsymbol{p}=\left[\begin{array}{llllll}P & 0 & 0 & 0 & 0 & 0\end{array}\right]^{T}$.

For a given element $e$, from (17) one has

$$
\delta \varepsilon_{E}=\frac{1}{J\left(X_{l}\right)} \frac{d J\left(x_{l}\right)}{d \boldsymbol{u}_{l}} \delta \boldsymbol{u}_{l},
$$

and noting that $(22)$ is valid for an arbitrary $\delta \boldsymbol{u}_{l}$, after some manipulations, it results

$$
\boldsymbol{f}_{l}=\int_{-1}^{1} \boldsymbol{B}_{o}^{T} \sigma_{E} J\left(X_{l}\right) A_{o} d \xi
$$

where

$$
\boldsymbol{B}_{o}=\frac{1}{2 J\left(X_{l}\right)}\left[\begin{array}{llllll}
-1 & 0 & 0 & 1 & 0 & 0
\end{array}\right]
$$

In order to map the internal force vector to the global system of reference used in (12), the rotation matrix $T$ is employed, leading to

$$
\boldsymbol{f}^{e}=\boldsymbol{T}^{T} \boldsymbol{f}_{l}
$$

where the upperscript $e$ in $f^{e}$ indicates that the element vector has its coordinates refered to the global system of reference,

$$
\boldsymbol{T}=\left[\begin{array}{cc}
\boldsymbol{\lambda} & \mathbf{0} \\
\mathbf{0} & \boldsymbol{\lambda}
\end{array}\right] \text { and } \boldsymbol{\lambda}=\left[\begin{array}{lll}
l_{1} & m_{1} & n_{1} \\
l_{2} & m_{2} & n_{2} \\
l_{3} & m_{3} & n_{3}
\end{array}\right]
$$

In (26) and (27), $l_{i}, m_{i}$ and $n_{i}$ are the direction cosines of the orthogonal local axes of the bar with respect to the $i$-th global axis. In practice, noting that in (24) just the first and the fourth matrix components are nonzero, only $l_{1}, m_{1}$ and $n_{1}$ need to be computed. 


\subsection{Tangent Stiffness Matrix}

From (13), the tangent stiffness matrix is calculated by differentiation of the residual with respect to the displacement vector set in the global reference system. If the external force vector is constant at each load step, the tangent matrix for each element is given by

$$
\boldsymbol{k}_{T}=\frac{d \boldsymbol{f}^{e}}{d \boldsymbol{u}^{e}}=\frac{d}{d \boldsymbol{u}^{e}}\left(\boldsymbol{T}^{T} \boldsymbol{f}_{l}\right)
$$

where $\boldsymbol{u}^{e}$ is the element nodal displacement vector with respect to the global reference system. Differentiation of $(25)$ yields

$$
\boldsymbol{k}_{T}=\underbrace{\frac{d \boldsymbol{T}^{T}}{d \boldsymbol{u}^{e}} \boldsymbol{f}_{l}}_{\boldsymbol{k}_{T 1}}+\underbrace{\boldsymbol{T}^{T} \frac{d \boldsymbol{f}_{l}}{d \boldsymbol{u}^{e}}}_{\boldsymbol{k}_{T 2}} .
$$

After some algebra, the term $\boldsymbol{k}_{T 1}$ can be expressed as (Stahlschmidt, 2013)

$$
\boldsymbol{k}_{T 1}=\int_{-1}^{+1} \boldsymbol{B}^{T} \boldsymbol{H} \boldsymbol{B} J\left(X_{l}\right) d \xi
$$

where

$$
\begin{gathered}
\boldsymbol{B}=\frac{1}{2 J\left(X_{l}\right)}\left[\begin{array}{cccccc}
-1 & 0 & 0 & 1 & 0 & 0 \\
0 & -1 & 0 & 0 & 1 & 0 \\
0 & 0 & -1 & 0 & 0 & 1
\end{array}\right] \\
\boldsymbol{H}=\frac{\sigma_{E} A_{o}}{\lambda}\left[\boldsymbol{I}-\frac{1}{\lambda^{2}} \boldsymbol{B} \boldsymbol{x}^{e} \boldsymbol{x}^{e T} \boldsymbol{B}^{T}\right]
\end{gathered}
$$

$\lambda=L / L_{0}$ is the stretch ratio, $\boldsymbol{I}$ is the identity matrix of order 3 , and $\boldsymbol{x}$ contains the nodal coordinates with respect to the global system of reference.

The term $\boldsymbol{k}_{T 2}$ of Eq. (29) can be developed and arranged as

$$
\boldsymbol{k}_{T 2}=\boldsymbol{T}^{T}\left[\int_{-1}^{+1} \boldsymbol{B}_{o}^{T} E \boldsymbol{B}_{o} J\left(X_{l}\right) d \xi\right] \boldsymbol{T}=\boldsymbol{T}^{T} \boldsymbol{k}_{T 2 l} \boldsymbol{T} .
$$

The global tangent stiffness $\boldsymbol{K}_{T}$ is obtained applying an assembly operator $\bigwedge_{e=1}^{n e l}$ which maps local to global degrees of freedom and sums the contribution of each element to the stiffness (or force vectors) of the whole structure (Hughes, 1986), i.e.

$$
\boldsymbol{K}_{T}=\bigwedge_{e=1}^{n e l} \boldsymbol{k}_{T}=\bigwedge_{e=1}^{n e l}\left(\boldsymbol{k}_{T 1}+\boldsymbol{k}_{T 2}\right)
$$


It is important to remark that in linear analyses, the term $\boldsymbol{k}_{T 1}$ vanishes and the linear equation (13) is exact, so the whole load can be applied in just one step $\left(\mu_{1}=1\right), \tilde{\boldsymbol{u}}_{1}^{0}=0$ and $\boldsymbol{R}_{1}\left(\tilde{\boldsymbol{u}}_{1}^{0}=0\right)=0$. Thus, the discretized equilibrium (13) particularizes to

$$
\begin{aligned}
& \boldsymbol{R}_{1}\left(\tilde{\boldsymbol{u}}_{1}^{1}=\boldsymbol{u}\right)=\boldsymbol{p}-\boldsymbol{f}\left(\tilde{\boldsymbol{u}}_{1}^{0}\right) \\
& \boldsymbol{R}_{1}\left(\tilde{\boldsymbol{u}}_{1}^{1}=\boldsymbol{u}\right)=\boldsymbol{R}_{1}\left(\tilde{\boldsymbol{u}}_{1}^{0}=\mathbf{0}\right)+\frac{d \boldsymbol{R}_{1}\left(\tilde{\boldsymbol{u}}_{1}^{0}=\mathbf{0}\right)}{d \tilde{\boldsymbol{u}}_{1}^{0}} \boldsymbol{u},
\end{aligned}
$$

or

$$
p=K u,
$$

where

$$
\boldsymbol{K}=\bigwedge_{e=1}^{n e l} \boldsymbol{k}_{T 2}, \quad \boldsymbol{p}=\bigwedge_{e=1}^{n e l} \boldsymbol{p}^{e} \quad \text { and } \quad \boldsymbol{f}=\bigwedge_{e=1}^{n e l} \boldsymbol{f}^{e}
$$

\section{SENSITIVITY ANALYSIS METHODS}

A brief review of the main aspects of each of the sensitivity methods considered in this work is given in this Section.

\subsection{Methods Based on Real Variables}

\section{Overall (Global) Finite Difference Method}

In linear problems, the displacement sensitivity can be approximated using forward finite differences by running the FEM code independently for unperturbed and perturbed design variables and performing a finite difference between the displacements obtained, i.e.,

$$
\frac{d \boldsymbol{u}(s)}{d s_{j}} \approx \frac{\boldsymbol{u}\left(\boldsymbol{s}+\Delta \boldsymbol{s}_{j}\right)-\boldsymbol{u}(\boldsymbol{s})}{\Delta s_{j}}=\left[\frac{\Delta \boldsymbol{u}(s)}{\Delta s_{j}}\right]_{F F D},
$$

where $s$ represents the design variables vector and $\Delta s_{j}$ is a perturbation in the $j$-th design variable

$$
\Delta s_{j}=\left[\begin{array}{llllll}
0 & 0 & \cdots & \Delta s_{j} & \cdots & 0
\end{array}\right]^{T} .
$$

Similarly, central finite differences are calculated by

$$
\left[\frac{\Delta \boldsymbol{u}(s)}{\Delta s_{j}}\right]_{C F D}=\frac{\boldsymbol{u}\left(s+\Delta \boldsymbol{s}_{j}\right)-\boldsymbol{u}\left(\boldsymbol{s}-\Delta \boldsymbol{s}_{j}\right)}{2 \Delta s_{j}} .
$$


In nonlinear problems, the calculation of the finite difference sensitivity follows the same procedure, except that displacements are evaluated at the $n$-th load step using (13) and

$$
\boldsymbol{u}_{n}=\sum_{i=1}^{n} \Delta \boldsymbol{u}_{i}
$$

Equations (41) and (43) are replaced by

$$
\left[\frac{\Delta \boldsymbol{u}_{n}(\boldsymbol{s})}{\Delta s_{j}}\right]_{F F D}=\frac{\boldsymbol{u}_{n}\left(\boldsymbol{s}+\Delta s_{j}\right)-\boldsymbol{u}_{n}(\boldsymbol{s})}{\Delta s_{j}}
$$

and

$$
\left[\frac{\Delta \boldsymbol{u}_{n}(\boldsymbol{s})}{\Delta s_{j}}\right]_{C F D}=\frac{\boldsymbol{u}_{n}\left(\boldsymbol{s}+\Delta s_{j}\right)-\boldsymbol{u}_{n}\left(\boldsymbol{s}-\Delta s_{j}\right)}{2 \Delta s_{j}},
$$

where the absence of the tilde over the displacement vector $u_{n}$ indicates that the converged value for the $n$-th load step is used in the computation.

\section{Semi-Analytical Method}

In the linear case, displacement sensitivity is obtained by differentiation of (37) with respect to the design variable $s_{j}$, which leads to

$$
\frac{d \boldsymbol{u}(s)}{d s_{j}}=\boldsymbol{K}^{-1}\left(\frac{d \boldsymbol{p}(s)}{d s_{j}}-\frac{d \boldsymbol{K}(s)}{d s_{j}} \boldsymbol{u}(\boldsymbol{s})\right)
$$

Evaluating the derivatives in the RHS exactly, leads to the so-called analytical method. On the other hand, if the same derivatives are approximated via finite differences, the semi-analytical sensitivity expression below is obtained

$$
\frac{d \boldsymbol{u}(s)}{d s_{j}} \approx \boldsymbol{K}^{-1}\left(\frac{\Delta \boldsymbol{p}(s)}{\Delta s_{j}}-\frac{\Delta \boldsymbol{K}(s)}{\Delta s_{j}} \boldsymbol{u}(s)\right) .
$$

Similarly, in the nonlinear case, the residual given by (13) is differentiated. If the derivatives in the RHS with respect to the design variable $s_{j}$ are calculated exactly, the analytical method results. On the other hand, if the same derivatives are approximated via finite differences, the semi-analytical expression is obtained

$$
\frac{d \boldsymbol{u}_{n}}{d s_{j}} \approx\left(\boldsymbol{K}_{T}^{n}\right)^{-1}\left[\frac{-\Delta \boldsymbol{R}_{n}\left(\boldsymbol{u}_{n}, \boldsymbol{s}\right)}{\Delta s_{j}}\right]=\left(\boldsymbol{K}_{T}^{n}\right)^{-1}\left[\frac{\Delta \boldsymbol{f}\left(\boldsymbol{u}_{n}, \boldsymbol{s}\right)}{\Delta s_{j}}-\mu_{n} \frac{\Delta \boldsymbol{p}(\boldsymbol{s})}{\Delta s_{j}}\right] .
$$


An important aspect to be observed is that the algebraic system given by Eq. (49) is linear. As there are no iterations to correct any inaccuracy in the solution, the use of the exact tangent matrix is mandatory. This is the reason that motivated the previous presentation of Section 2.3.

The numerical implementation of the semi-analytical procedure using both forward and central finite differences is shown in Figs. 3 and 4.

\subsection{Methods Based on Complex Variables}

A method for the evaluation of derivatives employing complex variables was developed in the 70 s (Lyness and Moler, 1967), and has been increasingly explored in several areas of engineering (Newman III et al., 1998; Martins et al., 2000; Burg and Newman III., 2001; Kawamoto et al., 2005; Mundstock and Marczak, 2009; Jin et al., 2009; Jin et al., 2010; Montoya et al., 2015). The method is extremely accurate, since when double-precision complex numbers are used, the smallest non-zero number that can be represented is $10^{-308}$ (Martins et al., 2000). It is also a promising alternative to deal with drawbacks present in finite differences and in the semi-analytical method based on real variables.

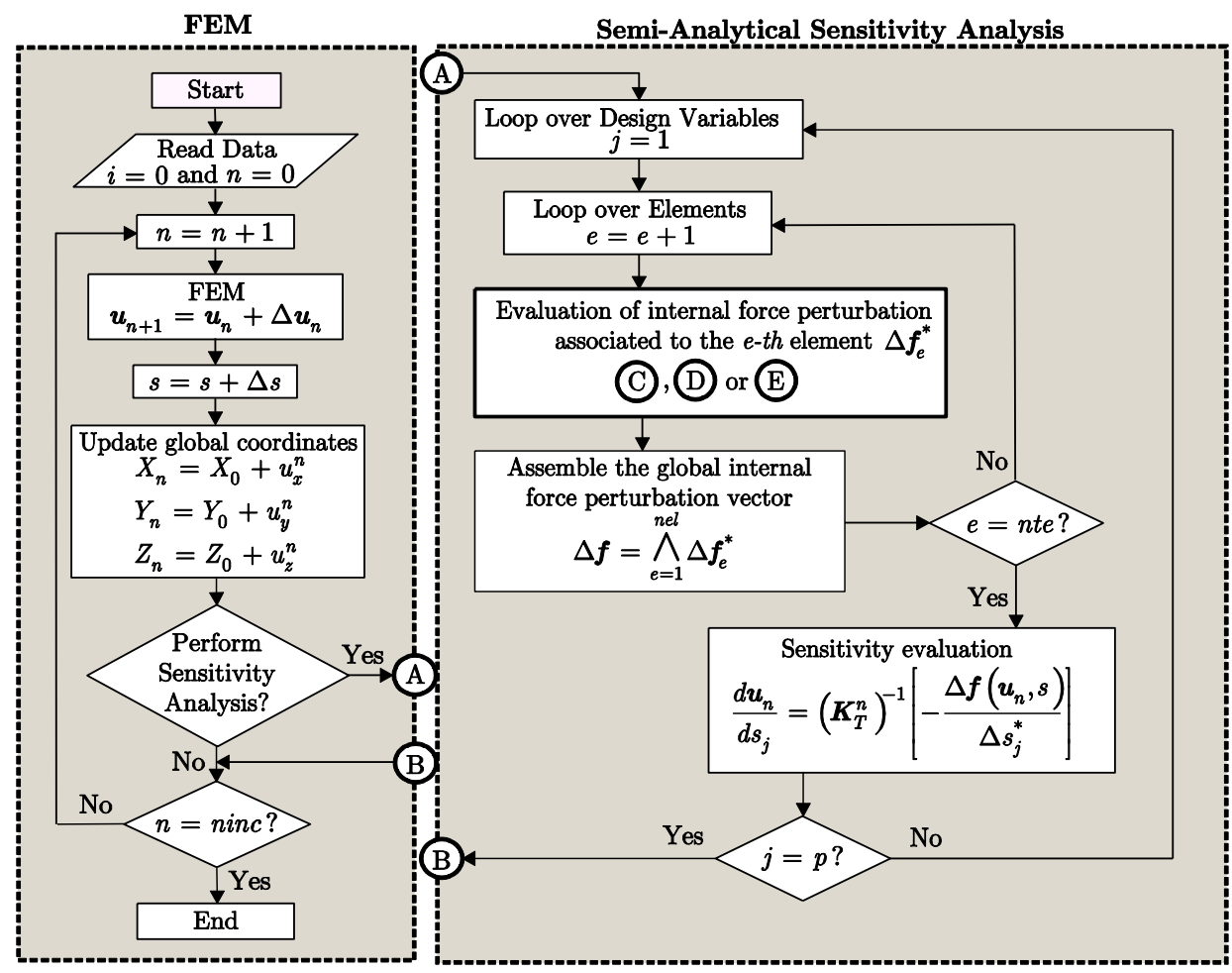

Figure 3: Calculation of displacement sensitivities via the semi-analytical method. Particularization for real or complex variables depends on the procedure that evaluates the internal force variation at the element level $\left(\Delta f_{e}^{*}\right)$. 


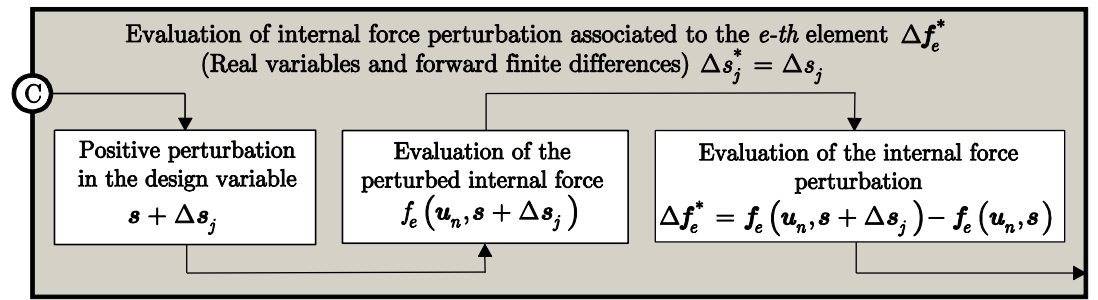

(a)

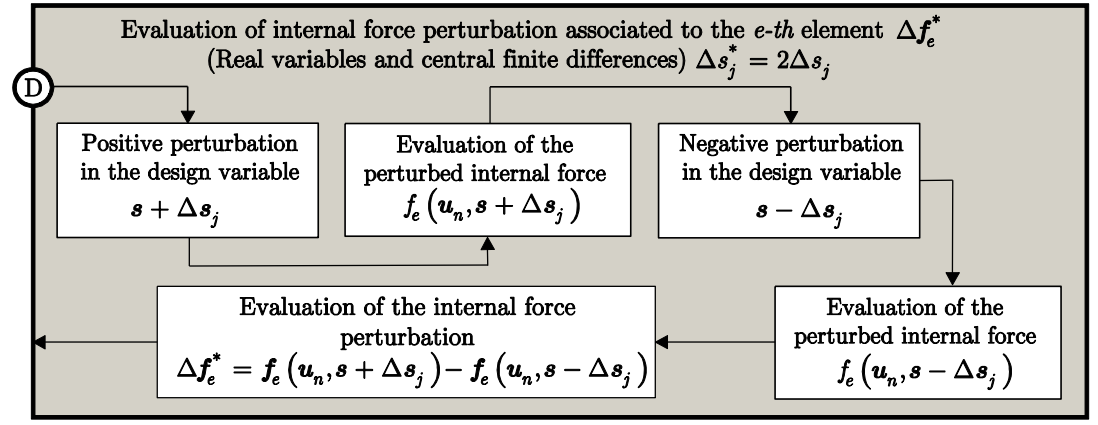

(h)

Figure 4: Details of the procedure adopted to evaluate the internal force variation (highlighted block in Fig. 3), in case of the RVSA method: (a) using FFD and (b) using CFD.

\section{Global Finite Difference Method with Complex Variables}

Following Lyness and Moler (1967) apud Mundstock (2006), the first derivative of a function $f(s)$ can be calculated by simple evaluation of the imaginary part of the perturbed function in a given point. To show this, let us expand the function $f(s)$ using a Taylor series,

$$
f(s+\Delta s)=f(s)+f^{\prime}(s) \Delta s+\frac{f^{\prime \prime}(s) \Delta s^{2}}{2}+\frac{f^{\prime \prime \prime}(s) \Delta s^{3}}{6}+\ldots
$$

Now, if instead of introducing the real perturbation $\Delta s$, this is replaced by an imaginary counterpart $i \Delta s$, the Taylor expansion gets the form

$$
f(s+i \Delta s)=f(s)+f^{\prime}(s) i \Delta s+\frac{f^{\prime \prime}(s)(i \Delta s)^{2}}{2}+\frac{f^{\prime \prime \prime}(s)(i \Delta s)^{3}}{6}+\ldots
$$

Using the fact that $i^{2}=-1$, the imaginary part of the Eq. (51) is

$$
\operatorname{Im}[f(s+i \Delta s)]=f^{\prime}(s) \Delta s-\frac{f^{\prime \prime \prime}(s) \Delta s^{3}}{6}+\ldots
$$

and the first order derivative of $f(s)$ is given by

$$
f^{\prime}(s)=\frac{\operatorname{Im}[f(s+i \Delta s)]}{\Delta s}+O\left(\Delta s^{2}\right) .
$$


It is important to observe that the sensitivity approximation given by (53) does not involve subtraction between two different (and close) values, a fact that produces error in the traditional finite difference method. Moreover, the remainder of the approximation in (53) is of order 2, while the traditional finite difference counterpart provides approximation of order 1. Approximation of order 2 means that the sensitivity converges quadratically with a decrease in the perturbation (Martins et al., 2000). These aspects enable the CVSA method to yield extremely accurate results even when tiny perturbations are applied.

\section{Semi-Analytical Complex Variable Method}

In spite of the advantages of the CVSA method mentioned in the foregoing, its direct use in the framework of a finite element code has an important disadvantage. The technique requires declaring a large number of global vectors and matrices (total number of degrees of freedom) as complex. Hence, large memory storage is required and a very much increased computational effort to perform algebraic operations result (Jin et al., 2010).

Consider now that in (47) the derivatives of the stiffness matrix and external loading with respect to the design variables are evaluated using a complex finite difference approach. Then the formulation of the CVSA method for linear problems is obtained, i.e.,

$$
\frac{d \boldsymbol{u}(\boldsymbol{s})}{d s_{j}} \approx \boldsymbol{K}^{-1}(s)\left(\frac{\operatorname{Im}\left(\boldsymbol{K}\left(\boldsymbol{s}+i \Delta \boldsymbol{s}_{j}\right)\right)}{\Delta s_{j}} \boldsymbol{u}(\boldsymbol{s})-\frac{\operatorname{Im}\left(\boldsymbol{p}\left(\boldsymbol{s}+i \Delta \boldsymbol{s}_{j}\right)\right)}{\Delta s_{j}}\right) .
$$

If in (49) the derivatives of the internal and external force vectors with respect to the design variables are approximated using the complex variable finite difference method, the formulation of the CVSA method for nonlinear problems is obtained, i.e.,

$$
\frac{d \boldsymbol{u}_{n}(\boldsymbol{s})}{d s_{j}} \approx\left(\boldsymbol{K}_{T}^{n}(\boldsymbol{s})\right)^{-1}\left(\frac{\operatorname{Im}\left(\boldsymbol{f}\left(\boldsymbol{u}_{n}, \boldsymbol{s}+i \Delta \boldsymbol{s}_{j}\right)\right)}{\Delta s_{j}}-\mu_{n} \frac{\operatorname{Im}\left(\boldsymbol{p}\left(\boldsymbol{s}+i \Delta \boldsymbol{s}_{j}\right)\right)}{\Delta s_{j}}\right) .
$$

Notice that in all the semi-analytical schemes, real or complex, the approximation of derivatives can be calculated inside the loop over elements and afterwards assembled to the global form, using the assembly operator defined in Section 2.4. Hence, the large storage requirement present in the global complex finite difference method is circumvented. No global complex vector or matrix is ever defined in the finite element code. It is worth highlighting that the gain in memory storage and in processing time (with respect to the global complex variable finite difference method) is much more pronounced and important in nonlinear cases than in linear ones. The numerical implementation of this approach is sketched in Figs. 3 and 5. 


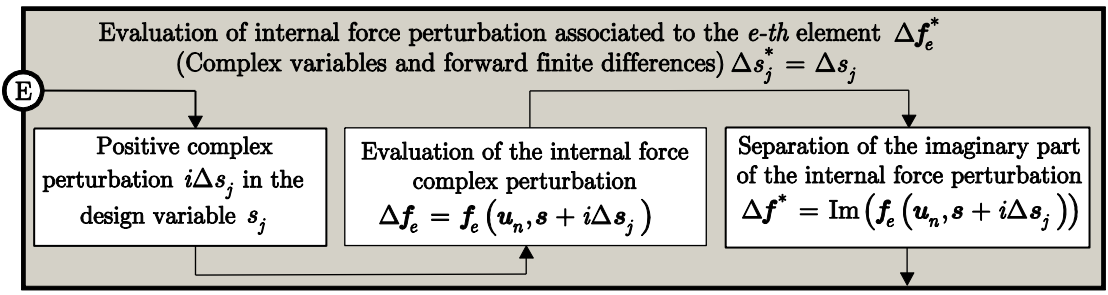

Figure 5: Detail of the procedure adopted to evaluate the internal force variation for the semi-analytical complex variable method (highlighted block in Fig. 3) using forward finite differences.

\section{EXAMPLE}

The finite element formulation and the sensitivity analysis methods detailed in Sections 2 and 3 were all implemented in a unique academic code, so that results could be compared fairly. A number of aspects related to a cantilever beam-like truss, subject to a point load on its free end (Barthelemy and Haftka, 1990) were studied in order to analyze the behavior of the CVSA method. For later reference, the term accuracy stability will be used for the range of perturbation factors where the error is continuously equal or below $0.1 \%$.

Barthelemy and Haftka (1990) analyzed the behavior of the strain energy sensitivity with respect to geometrical parameters in a beam modeled using bar finite elements. The structure was built-up of square cells, each containing 5 bars, as depicted in Fig. 6 . The number of cells considered in their work varied between 1 and 20, and the sensitivity was evaluated using the RVSA method and overall finite differences.

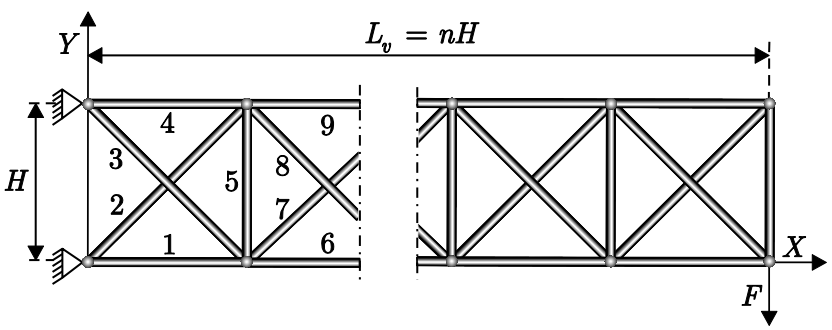

Figure 6: Beam modeled using bar finite elements.

In the present research work, a different but similar problem is analyzed and the displacement sensitivities with respect to shape and material design variables are studied. The number of cells $(n)$ ranges from 1 to 60 and the dimension $H$ adopted is $100 \mathrm{~mm}$. Following Barthelemy and Haftka (1990), the area of internal elements $\left\{2+5 n\right.$ and $\left.3+5 n, n \in \mathbb{Z}_{+}\right\}$is made 125 times larger than that of the external elements, $A_{0}=7 \mathrm{~mm}^{2}$. This geometrical condition enforces deformation to be dominated by rigid rotations, thus configuring an appropriate situation for evaluating the shape sensitivity pathology usually present in RVSA methods. The value adopted for the Young modulus is $E=2.1 \times 10^{5} \mathrm{MPa}$ and the vertical load is prescribed to $F=2.0 \times 10^{-5} \mathrm{~N}$. Linear and geometrical nonlinear formulations are studied and the Newton-Raphson (Crisfield, 1991) is used to solve this Latin American Journal of Solids and Structures 12 (2015) 980-1005 
problem. The quadratically convergence property was verified, a priori, validating the correct implementation of the tangent matrix with the exact linearization developed in Section 2.3.

\section{Displacement Sensitivity with Respect to a Shape Parameter}

In this case, the design variable is the length of the beam $L_{v}$. For a given perturbation, all the horizontal nodal coordinates are updated proportionally according to

$$
\Delta s=\frac{\boldsymbol{x}}{L_{v}}\left(\varphi L_{v}\right)
$$

where $\boldsymbol{x}$ stands for the vector of horizontal nodal coordinates, and $\varphi$ represents the perturbation factor used, which varies from $10^{-30}$ to $10^{-1}$.

\section{Displacement Sensitivity with Respect to a Material Parameter}

The behavior of the described semi-analytical methods on a material parameter is studied in this Section. The Young modulus of element 1 is selected as the material parameter to be perturbed and the perturbation factor $\varphi$ is varied from $10^{-30}$ to $10^{-1}$. The perturbation applied is, therefore

$$
\Delta s=\varphi E^{(1)} .
$$

The results obtained for both types of parameters (shape and material) are shown first for the linear and then for the geometrically nonlinear formulations. The linear semi-analytical results obtained make use of same equations (49) and (55) employed for nonlinear analyses, yet introducing the assumptions discussed in (35) to (40).

\subsection{Linear Formulation}

In linear analyses, it makes no sense to apply more than one load step. Thus, the $L_{1}$ norm of the sensitivity vector and its associated relative error, are respectively given by

$$
\bar{S}_{L}=\sum_{j=1}^{n t n}\left(\left|\frac{d u_{j}}{d s}\right|+\left|\frac{d v_{j}}{d s}\right|\right) \text { and } \quad \bar{E}_{L}=100 \times \frac{\bar{S}_{L}-r e f}{r e f}
$$

These are global measures, since they include all the displacement sensitivity components. The reference values ref, are calculated using the CVSA method with $\varphi=10^{-30}$, as shown in Table 1 . 


\begin{tabular}{ccccc}
\hline \multirow{2}{*}{$\begin{array}{c}\text { Number of } \\
\text { cells }\end{array}$} & \multicolumn{2}{c}{$\begin{array}{c}\text { Reference value }(\text { ref) for } \\
\text { shape design variable }\end{array}$} & \multicolumn{2}{c}{$\begin{array}{c}\text { Reference value }(\text { ref) for } \\
\text { material design variable }\end{array}$} \\
\cline { 2 - 5 } & Linear & Nonlinear & Linear & Nonlinear \\
\hline 1 & 0.684889949047208 & 15.5802088815197 & $8.606282753999 \times 10^{-5}$ & $1.798827999570 \times 10^{-3}$ \\
5 & 37.4288195070117 & 421.829947620554 & $8.747946457966 \times 10^{-3}$ & $5.590497658866 \times 10^{-2}$ \\
10 & 250.705670225946 & 976.875382196364 & $6.770326423325 \times 10^{-2}$ & 0.149576377081562 \\
15 & 792.780706818079 & 1506.91861071136 & 0.225461613218021 & 0.251556225643772 \\
20 & 1816.71515440133 & 2028.33519556715 & 0.530612244996758 & 0.356608475045943 \\
25 & 3475.57023696911 & 2543.65887025360 & 1.03174603202345 & 0.462780200258804 \\
30 & 5922.31643772276 & 3054.97736673712 & 1.77745383906433 & 0.569449739327190 \\
35 & 9305.58180211248 & 3563.67821297505 & 2.81632653340653 & 0.676402535860982 \\
40 & 13770.8071289674 & 4070.51451568051 & 4.19695496460214 & 0.783570054554749 \\
45 & 19466.4871126662 & 4576.23176110257 & 5.96793002241570 & 0.890890846731269 \\
50 & 26542.9427280356 & 5081.06219110005 & 8.17784257766088 & 0.998304770766448 \\
55 & 35151.4911692424 & 5585.31781074625 & 10.8752834405527 & 1.10585057564113 \\
60 & 45444.0315380609 & 6088.95385961914 & 14.1088435927454 & 1.21342245357349 \\
\hline \hline
\end{tabular}

Table 1: Reference values for the beam modeled with bar elements.

Values obtained using the CVSA method and a perturbation factor $\varphi=10^{-30}$.

\subsubsection{Shape Design Variable}

For 5 cells, Fig. 7 (left) shows that the use of the CFD-RVSA method provides a wider range of accuracy stability than its FFD counterpart. In fact, the FFD scheme shows stable accuracy in the range $\varphi=10^{-12}$ to $10^{-6}$, while the CFD-RVSA does so in the range $\varphi=10^{-13}$ to $10^{-3}$. Overall finite difference schemes shows a distinct accuracy stability region if compared to semi-analytical methods, $\varphi=10^{-10}$ to $10^{-2}$ for CFD-OFD and $\varphi=10^{-9}$ to $10^{-3}$ for FFD-OFD. The CVSA procedure showed nearly exact results except for very large perturbations ( $\varphi=10^{-2}$ or more), where the sensitivity approximation corresponds no longer to a derivative but to a secant.

For 60 cells, Fig. 7 (right) shows that the accuracy stability range for all methods is reduced, when compared to the situation with 5 cells. The RVSA methods show stable accuracy for perturbation factors from $\varphi=10^{-13}$ to $10^{-5}$ for CFD and $\varphi=10^{-12}$ to $10^{-8}$ for FFD, respectively. The overall finite differences procedures shows good values for large perturbations, but a narrower range of accuracy stability than any of the real semi-analytical methods, between $\varphi=10^{-5}$ to $10^{-2}$ for CFD-OFD and $\varphi=10^{-5}$ to $10^{-3}$ for FFD-OFD, respectively. The CVSA method only shows nonaccurate results for perturbation factors larger than $\varphi=10^{-5}$. In fact, the CVSA method remained accurate for very small perturbations, including $\varphi=10^{-300}$ (minimum tested), where it presented a relative difference of $-3.56 \times 10^{-9} \%$ considering the reference at $\varphi=10^{-30}$. 

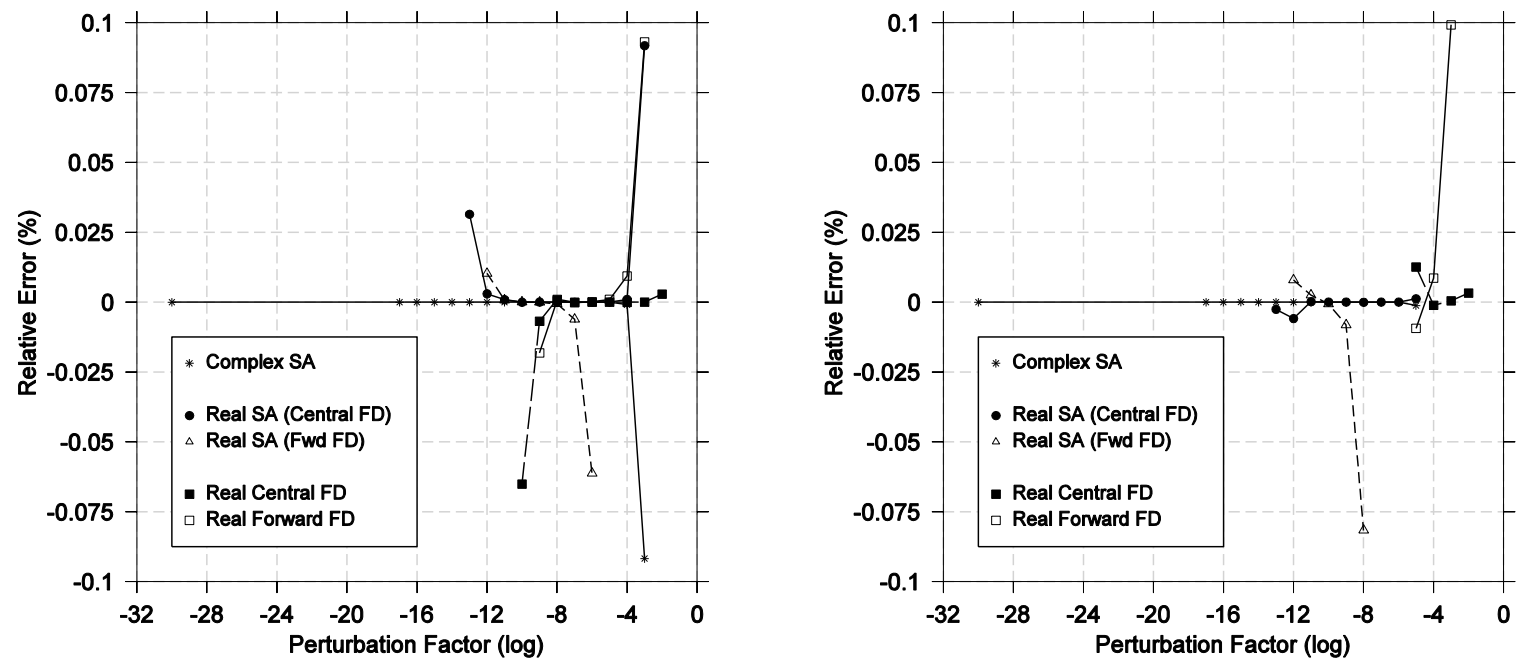

Figure 7: $\bar{E}_{L}$ for shape design variable: 5 cells (left) and 60 cells (right).

As seen in Fig. 7, the accuracy stability range for the shape sensitivity depends on the method employed and on the number of cells. A summary of the results obtained for semi-analytical methods for different number of cells is displayed in Fig. 8 and the particular cases for $\varphi=10^{-16}, 10^{-10}$ and $10^{-2}$ are studied in deeper detail in Figs. 9 and 10.

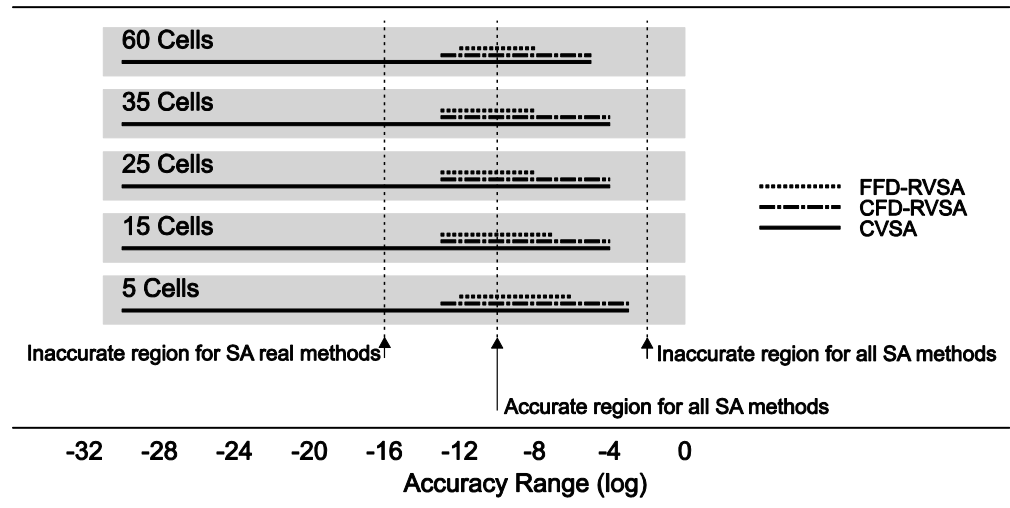

Figure 8: $\bar{S}_{L}$ for shape design variable: accuracy ranges.

Figure 9 (left) shows the error behavior $\left(\bar{E}_{L}\right)$ versus the number of cells $(n)$ for a perturbation factor $\varphi=10^{-16}$. It can be seen in Fig. 8 that this perturbation value lies in an inaccurate region for all the real semi-analytical methods. Figure 9 (left) shows that both RVSA methods oscillate around the reference value and high errors are involved. On the other hand, the CVSA method remains accurate and almost exact for the whole domain of analysis. 

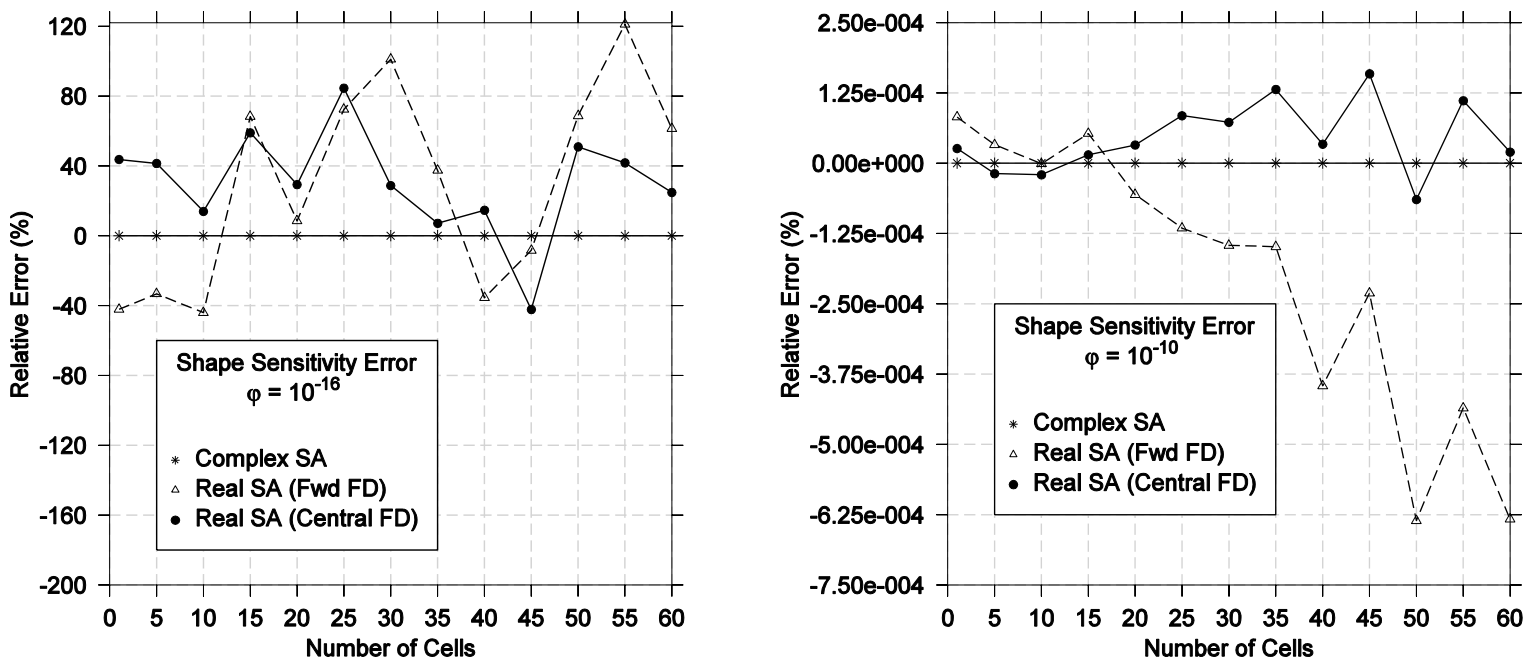

Figure 9: $\bar{E}_{L}$ versus the number of beam cells $n$. Results for $\varphi=10^{-16}$ in the left (inaccurate region for SA real methods), and for $\varphi=10^{-10}$ in the right (accurate region for all SA methods).
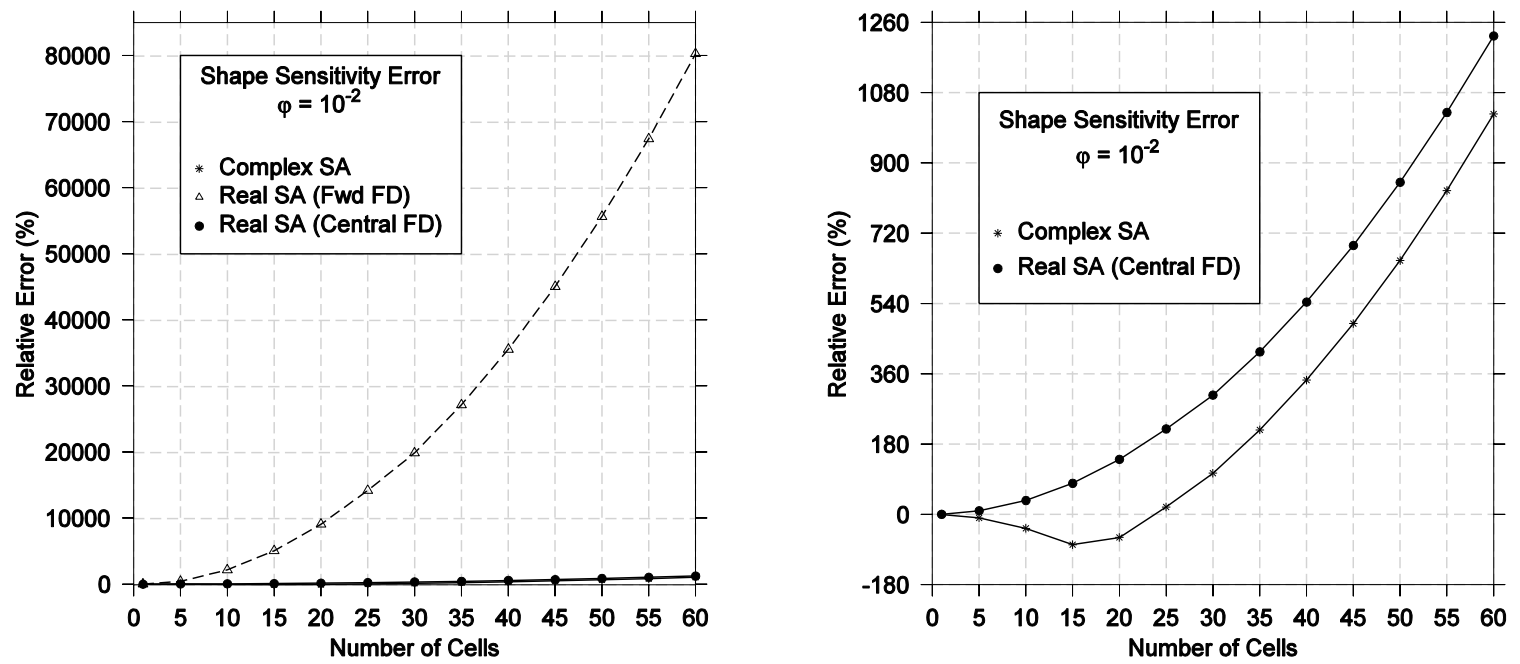

Figure 10: $\bar{E}_{L}$ for shape sensitivity versus the number of beam cells. Results for $\varphi=10^{-2}$, equivalent to $1 \%$ of effective perturbation (inaccurate region for all SA methods).

Figure 9 (right) presents the error behavior for the RVSA and CVSA methods when a perturbation factor $\varphi=10^{-10}$ is applied. Figure 8 evinces that this perturbation value belongs to a region where all the SA methods are accurate. Indeed, notice that the vertical scale in Fig. 9 (right) comprehends a very small range.

As mentioned in the beginning of Section 4, this example is formulated in order to favor the presence of the pathology related to rigid rotations. Figure 10 shows how the error evolves with an Latin American Journal of Solids and Structures 12 (2015) 980-1005 
increasing number of cells when the perturbation factor equals $\varphi=10^{-2}$ (perturbation value equal to $1 \%$ of the beam's length). As mentioned, in this region all the SA methods are inaccurate.

Clearly, the FFD-RVSA method shows errors one order of magnitude higher than the CVSA and the CFD-RVSA counterparts. However, the rate of error growth of the CVSA method is 1.25 times higher, compared to the FFD-RVSA alternative. The latter presents a quadratic growth, in accordance to the literature. For completeness, it is verified that the CFD-RVSA method shows an error growth rate of 2.388. The CVSA method leads to absolute errors below those resulting from application of the CFD-RVSA, in the whole domain of analysis.

\subsubsection{Material Design Variable}

The displacement sensitivity with respect to the Young modulus behaves in a stable accurate manner for a wide range of perturbation factors. All the RVSA schemes show stable accuracy for $\varphi=10^{-12}$ to $10^{-1}$. For any number of cells, the OFD methods always provide a narrower stability accuracy range than the RVSA. For decreasing perturbation factors, it is also verified that the OFD always loses accuracy before the RVSA. On the other hand, for any perturbation factor in the range $\varphi=10^{-30}$ to $10^{-1}$, the CVSA presents almost exact results for any number of cells (maximum absolute error value equal to $6.9 \times 10^{-12} \%$ ). Accuracy of results in this case occurs even for perturbation factors as low as $10^{-300}$.

\subsection{Nonlinear Formulation}

The problem depicted in Fig. 6 is analyzed again, this time considering large displacements and rotations, so that nonlinear incremental analyses become necessary. Fifty equal load steps are applied in all the analyses, using the full Newton-Raphson Method. Displacement sensitivities with respect to the shape and material parameters are computed according to the $L_{1}$ norm, yielding the $\bar{S}_{N L}$ measure given in (60). The corresponding relative error value $\bar{E}_{N L}$ is calculated using (61).

$$
\bar{S}_{N L}=\sum_{i=1}^{n i n c} \sum_{j=1}^{n t n}\left(\left|\frac{d u_{j}}{d s}\right|+\left|\frac{d v_{j}}{d s}\right|\right) \text { and } \quad \bar{E}_{N L}=100 \times \frac{\bar{S}_{N L}-r e f}{r e f}
$$

Equations (60) and (61) are analogous to (58) and (59), now including a sum over all the incremental steps, thus taking into consideration the sensitivity history along the loading application. Equation (61) uses the reference values ref, shown in Table 1.

\subsubsection{Shape Design Variable}

According to Fig. 11, the CFD-RVSA method shows stable accurate values for perturbation factors $\varphi=10^{-13}$ to $10^{-3}$ for 5 cells and $\varphi=10^{-14}$ to $10^{-3}$ for 60 cells. The FFD counterpart presents a narrower range of stable accuracy for any number of cells. This range comprehends $\varphi=10^{-13}$ to $10^{-6}$ 
for 5 cells and $\varphi=10^{-14}$ to $10^{-7}$ for 60 cells. As a rule, the OFD methods provide a larger stable accuracy range and lower relative errors when compared to the RVSA procedure.

The CFD-OFD is stable accurate for $\varphi=10^{-13}$ to $10^{-2}$ in the case of 5 cells and $\varphi=10^{-14}$ to $10^{-1}$ for 60 cells. The FFD counterpart shows stable accuracy between $\varphi=10^{-12}$ and $10^{-3}$, and between $\varphi=10^{-13}$ and $10^{-1}$ for 5 and 60 cells, respectively. This Figure illustrates clearly that the CVSA method keeps stable accurate for all the perturbation factors lower than $\varphi=10^{-3}$. Although not shown, this feature is observed for perturbation factors as low as $\varphi=10^{-300}$. The relative difference between the values obtained for $\varphi=10^{-30}$ and $\varphi=10^{-300}$ is $-1.02 \times 10^{-10_{\%}}$.
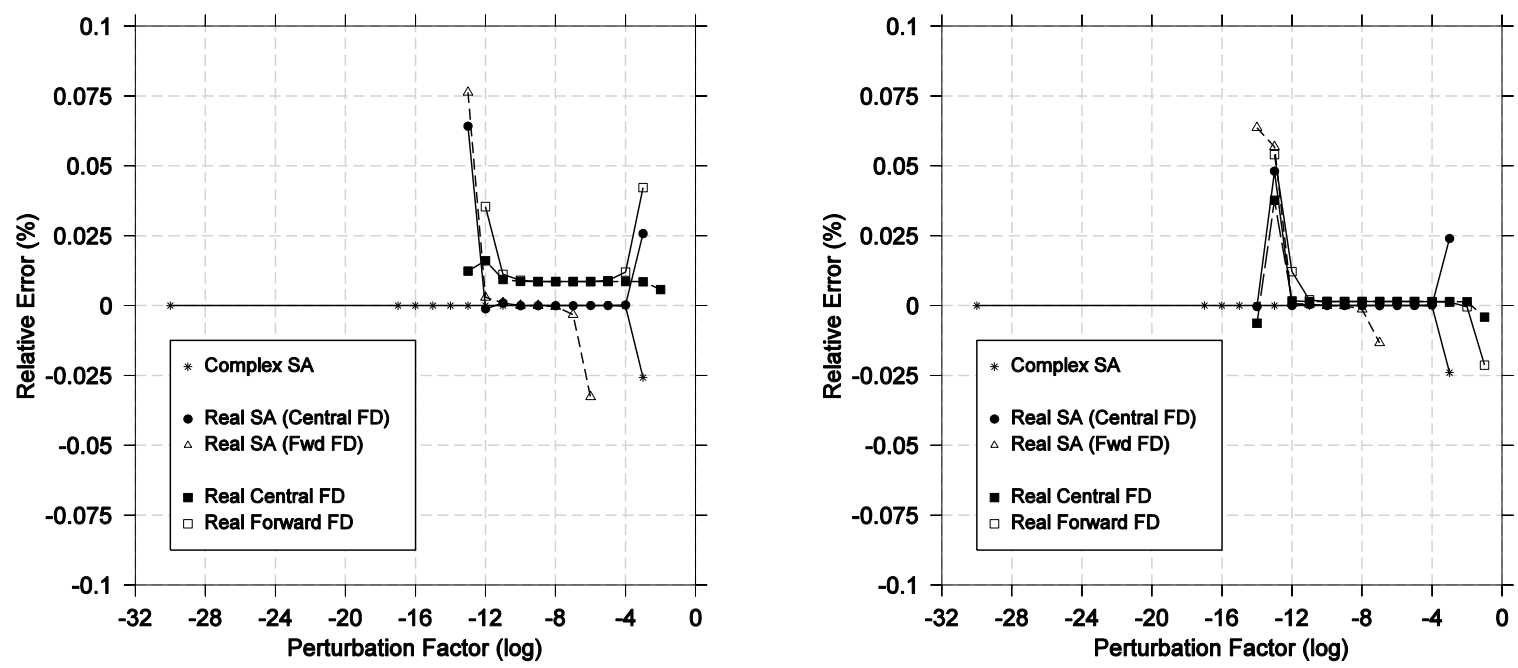

Figure 11: $\bar{E}_{N L}$ for shape design variable: 5 cells (left) and 60 cells (right).

A summary of the stable accuracy range presented by the semi-analytical procedures is given in Fig. 12. Figures 13 and 14 show the error evolution pattern for increasing number of cells in three selected cases: $\varphi=10^{-16}$ (inaccurate region for $\mathrm{SA}$ real methods), $\varphi=10^{-10}$ (accurate region) and $\varphi=$ $10^{-2}$ (inaccurate region).

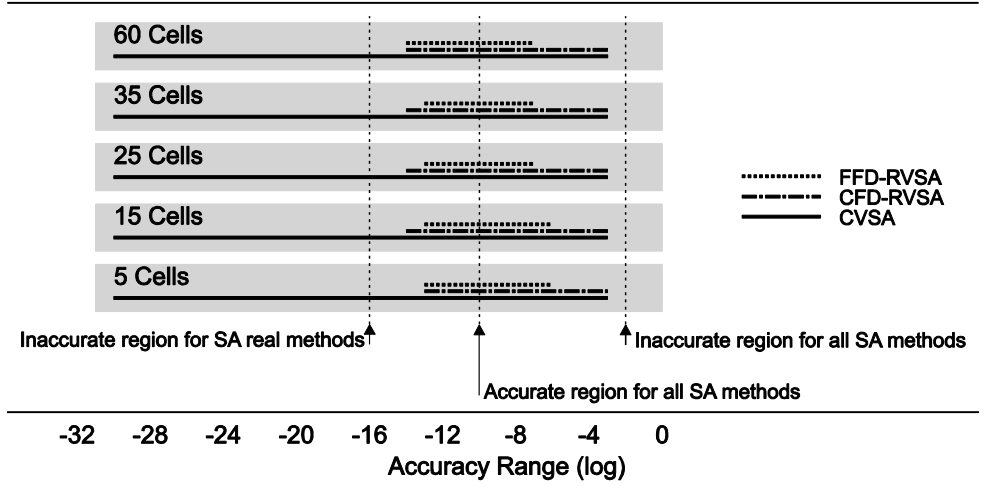

Figure 12: $\bar{S}_{N L}$ for shape design variable: accuracy ranges. 
Figure 13 (left) shows the error evolution for $\varphi=10^{-16}$. While the RVSA methods show high errors in the whole domain of analysis, the CVSA alternative shows absolute errors below $7.12 \times 10^{-11} \%$. Figure 13 (right) shows the error behavior for $\varphi=10^{-10}$. In this case, all the semi-analytical methods are accurate. Both RVSA procedures yield results that oscillate around the reference value, showing a maximum absolute error of $1.72 \times 10^{-4} \%$ (for 1 cell). Once more, the CVSA method presents accuracy, with absolute errors below $7.59 \times 10^{-11}$.
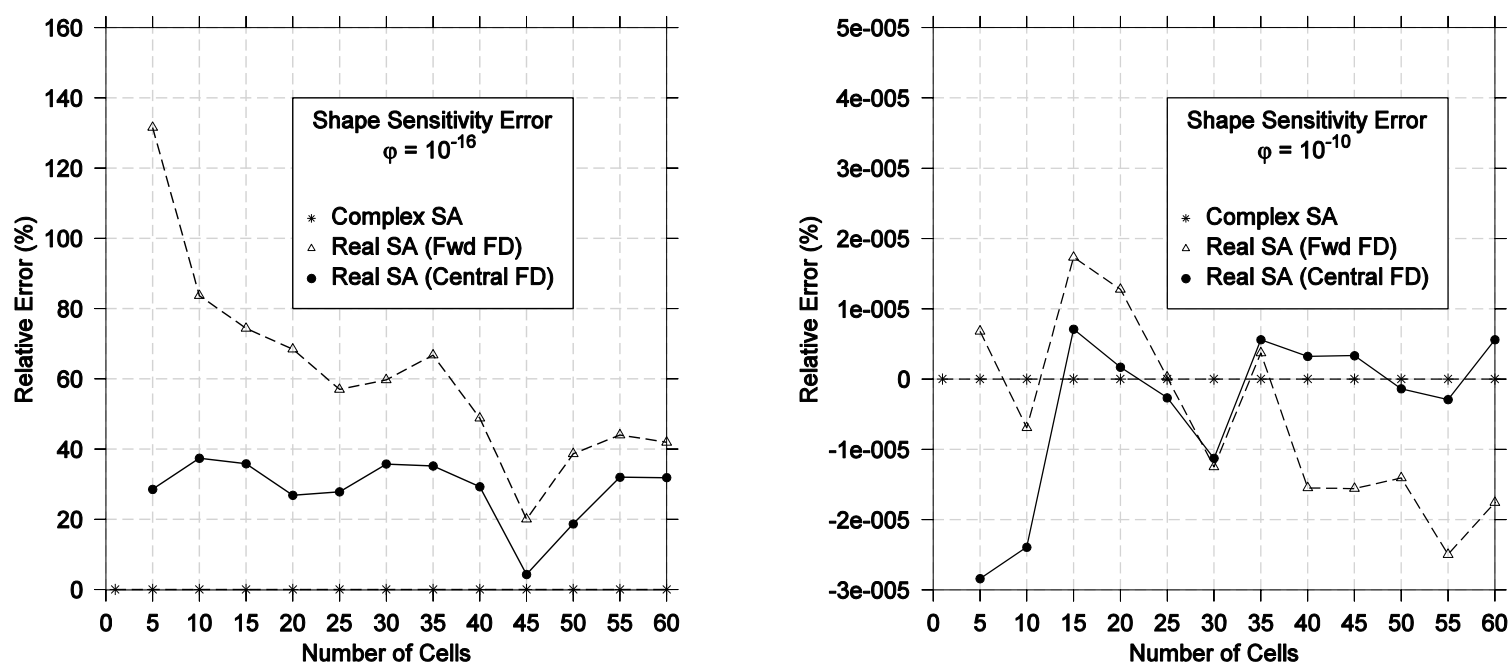

Figure 13: $\bar{E}_{N L}$ for shape sensitivity versus the number of beam cells. Results for $\varphi=10^{-16}$ in the left (inaccurate region for SA real methods) and the results for $\varphi=10^{-10}$ in the right (accurate region for all SA methods).
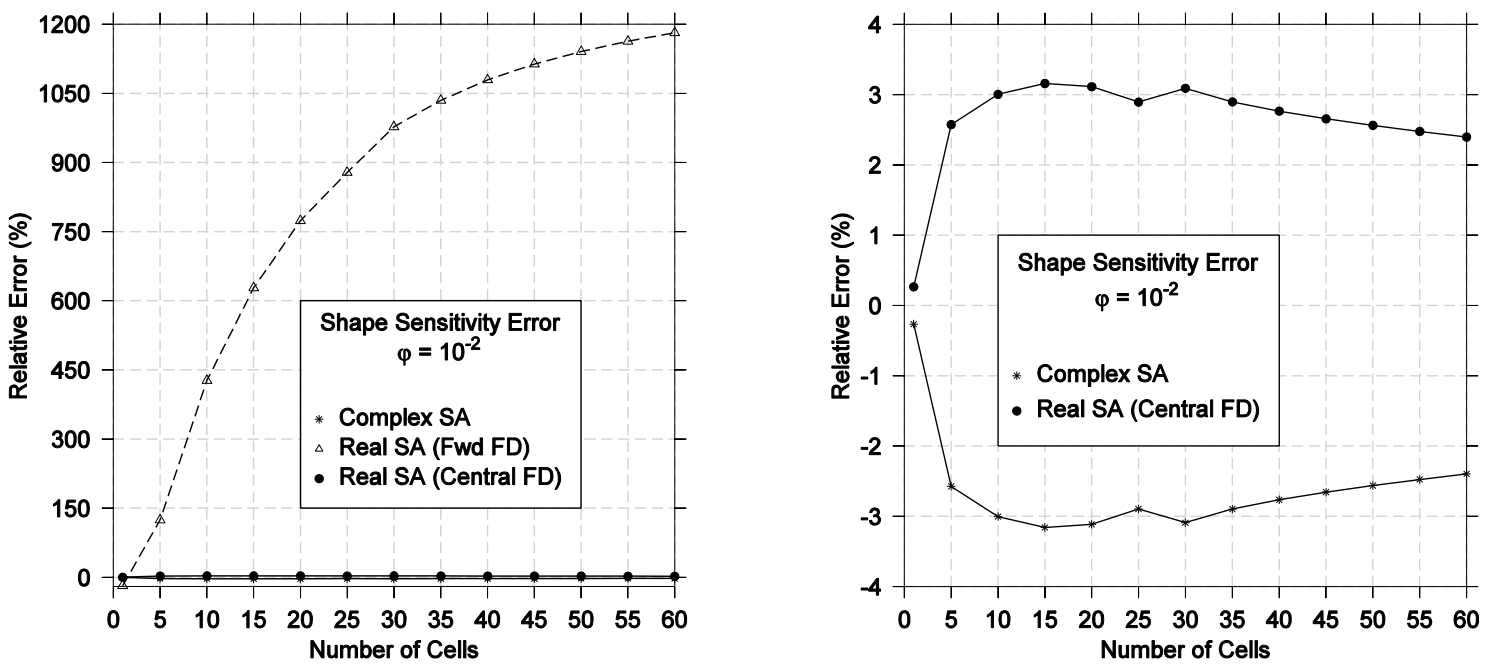

Figure 14: $\bar{E}_{N L}$ for shape sensitivity versus the number of beam cells. Results for $\varphi=10^{-2}$ equivalent to $1 \%$ of effective perturbation (inaccurate region for all SA methods). 
Figure 14 depicts the error evolution for an increasing number of cells when $\varphi=10^{-2}$, corresponding to an effective perturbation of $1 \%$ in the length of the beam. This is a moderately large perturbation, where typically the pathology associated to rigid rotations shows up. Indeed, the Figure evinces errors over $10^{3} \%$ when the FFD-RVSA is applied for more than 30 cells. Remarkably, the CVSA method shows absolute errors in the order of $2.5 \%$ to $3.5 \%$ for any number of cells, which is acceptable provided that the size of the perturbation applied. In accordance to the pathology behavior reported for the linear case in Fig. 10, in the nonlinear case the FFD-RVSA presents growing errors as the number of cells increases. An interesting fact is that in opposition to what happens in the linear case, the error's growth rate decreases for increasing number of cells. This can be explained by the fact that up to approximately 30 cells a considerable number of cells suffer rigid rotations (see Fig.15). Afterwards, bending begins to lose importance as the deformation becomes stretchdominated and the pathology error approaches a constant threshold. This phenomenon is not so strongly verified in the CVSA method, and diminishes as the perturbation factor decreases. In order to make this point even clearer, Figures 16 and 17 show the evolution of the incremental sensitivity errors, now defined nodally according to

$$
\begin{gathered}
\bar{S}_{\text {node }}^{i}=\left(\left|\frac{d u_{\text {node }}}{d s}\right|+\left|\frac{d v_{\text {node }}}{d s}\right|\right)_{i}, \\
A E_{\text {node }}^{i}=\left|\bar{S}_{\text {node }}^{i}-r e f_{\text {node }}\right| \text { and } R E_{\text {node }}^{i}=100 \times \frac{\bar{S}_{\text {node }}^{i}-r e f_{\text {node }}}{\text { ref }} .
\end{gathered}
$$
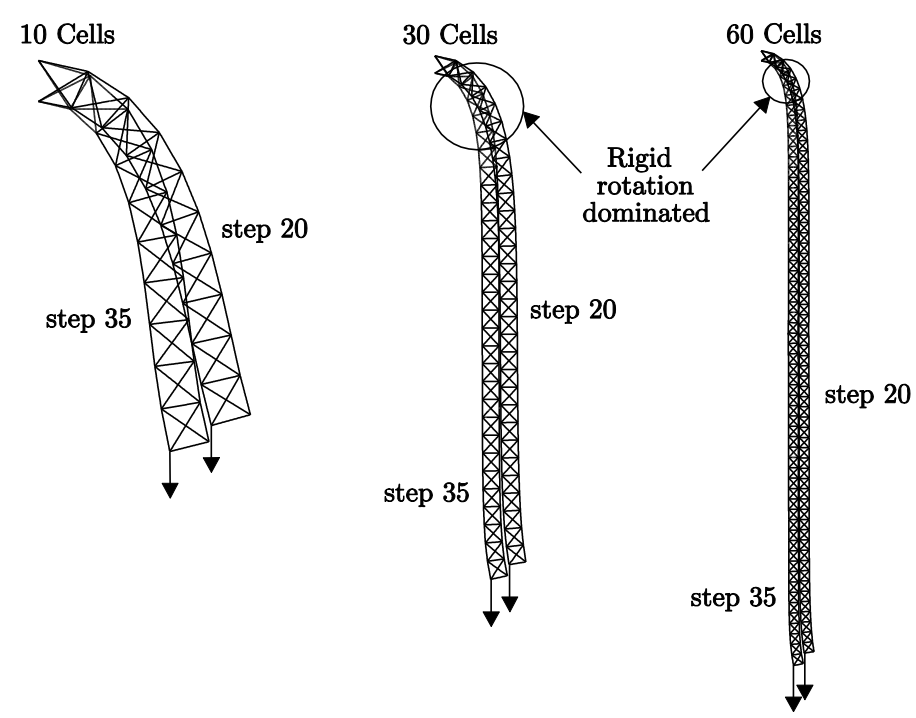

Figure 15: Bending-dominated regions for a beam modeled using bar finite elements for 5, 30 and 60 cells.

Notice that Node 14 is in a region subjected to bending strains and rotations, while node 62 suffers almost only rigid rotation and then stretching. Figures 16 and 17 show the evolution of the incremental errors for the RVSA and CVSA. As the latter admits tiny perturbation factors in a very 
stable manner, it is no problem to apply, for example, $\varphi=10^{-29}$, a value not supported by the RVSA. In this situation, the maximum absolute and relative errors for node 62 are approximately $5.9 \times 10^{-12}$ and $3.0 \times 10^{-10}$, respectively. In other words, the pathology is eliminated.
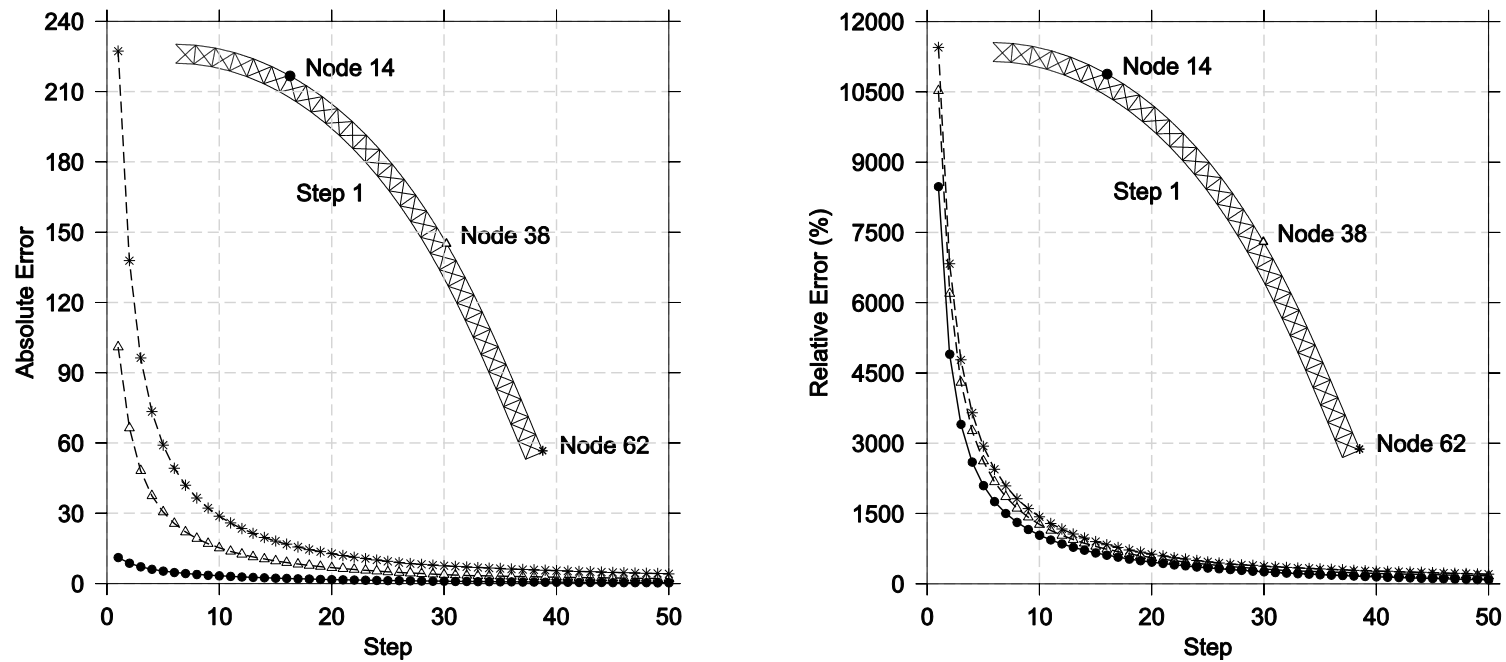

Figure 16: Absolute and relative errors for nodes subjected to severe, intermediate and mild rigid rotations (nodes 62,38 and 14). Results for the RVSA method and $\varphi=10^{-2}$.
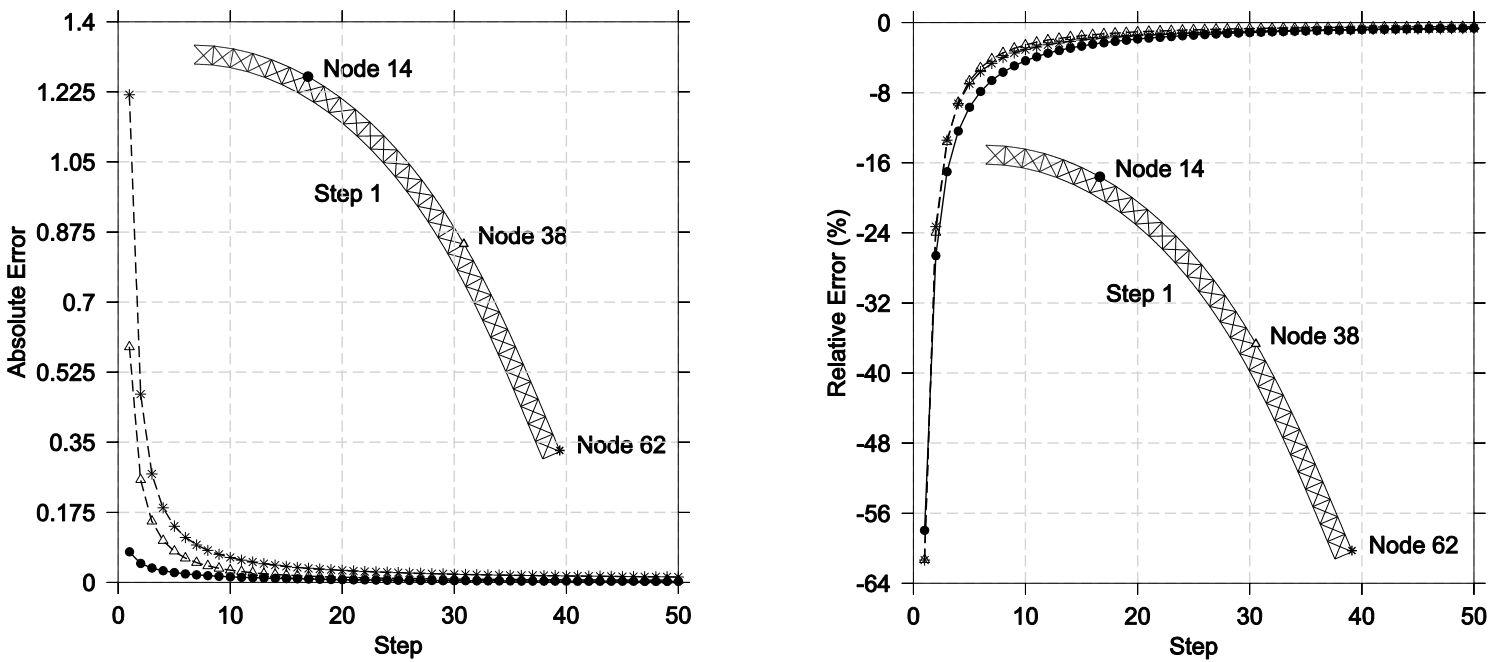

Figure 17: Absolute and relative errors for nodes subjected to severe, intermediate and mild rigid rotations (nodes 62,38 and 14). Results for the CVSA method and $\varphi=10^{-2}$.

\subsubsection{Effects of the Tolerance and Perturbation Factor on the Sensitivity}

In this work, it has been verified that the tolerance applied to the iterative solution of the nonlinear equations (13) affects strongly the accuracy of the displacement sensitivities. The same holds for the size of the perturbation factor. In order to show this effect, the clamped beam made of 60 cells has 
been taken for reference. The study has been made for the CVSA and FFD-RVSA methods, and the results have been compared to the CFD-OFD. In the analyses performed $\bar{S}_{N L}$ is particularized for the loaded node (bottom node of the free end) and current load step (increment) and thus, renamed as $\hat{S}_{N L}$.
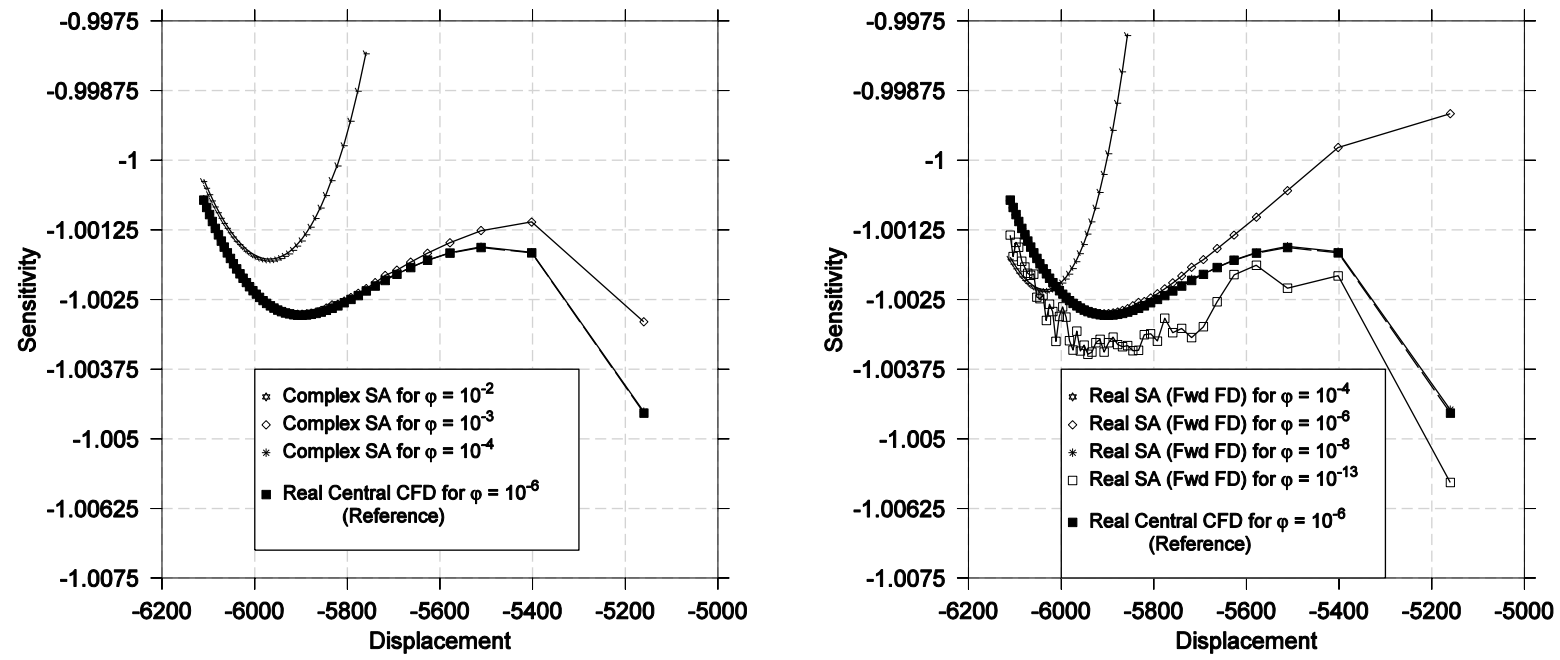

Figure 18: $\hat{S}_{N L}$ evolution with respect to the vertical displacement (tolerance $\left.=10^{-9}\right)$. CVSA (left) e FFD-RVSA (right).
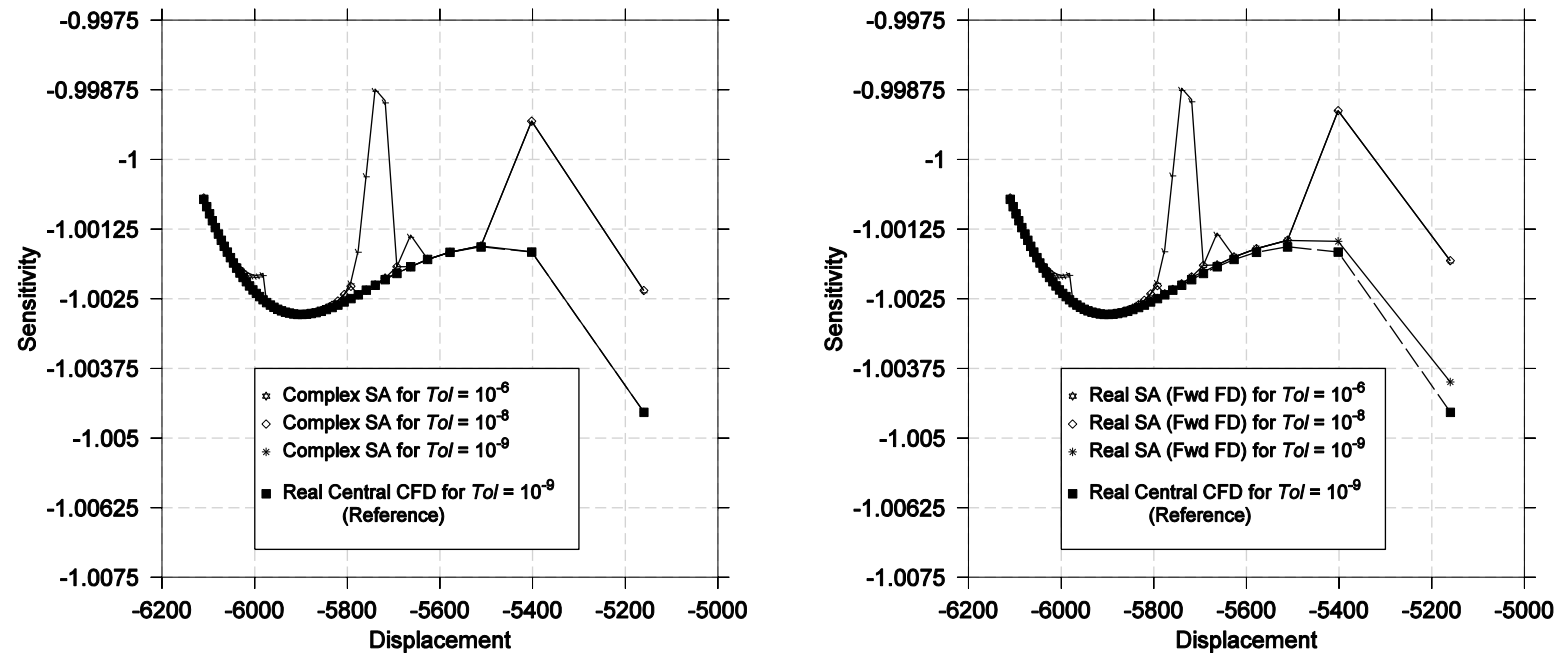

Figure 19: $\hat{S}_{N L}$ evolution with respect to the vertical displacement $\left(\varphi=10^{-7}\right)$. CVSA (left) and FFD-RVSA (right).

Figure 18 displays the evolution of the sensitivity measure $\hat{S}_{N L}$ versus the vertical displacement of the loaded node as the beam deforms. Each curve stands for a different perturbation factor, keeping Latin American Journal of Solids and Structures 12 (2015) 980-1005 
a fixed $10^{-9}$ tolerance in the nonlinear iterative process. In Fig. 18 (left) it can be seen that for $\varphi=$ $10^{-2}$, the CVSA curve is far from the correct behavior, whereas for $\varphi \leq 10^{-4}$ the curve matches the reference. Figure 18 (right) shows the influence of the perturbation factor on the results given by the FFD-RVSA for the same fixed tolerance. In this case only for $10^{-12} \leq \varphi \leq 10^{-8}$ the sensitivity curves reproduce the reference pattern. Clearly, all the curves show larger errors in the first load steps (lower displacements, at the right of the graphs), where rigid rotations affect more elements and the pathology contaminates the results.

As the perturbation factor $\varphi=10^{-7}$ was shown to lead to low errors for both CVSA and FFDRVSA, this value is kept constant for different imposed tolerances in the iterative solution of (13). Figure 19 displays the resulting curves for the CVSA (left) and FFD-RVSA (right) methods. The sensitivity curves generated by the CVSA using tolerance values equal to $10^{-8}$ and $10^{-9}$ are practically indistinguishable, except for the first two iterations. The FFD-RVSA method shows a similar behavior, however the curves obtained for tolerances equal to $10^{-8}$ and $10^{-9}$ visually differ from each other in the first 8 iterations. Tolerance values below $10^{-9}$ could not be evaluated due to machine accuracy.

\subsubsection{Material Design Variable}

For the material design variable, the sensitivity and error measures given by (60) and (61) are employed. Perturbations in the material parameter provided that a large range of stable accuracy in the analyzed cases. It was observed that all the real semi-analytical methods resulted in stable accurate values for perturbation factors ranging from $\varphi=10^{-14}$ to $10^{-1}$. The OFD methods showed slightly lower stable accuracy compared to the ranges achieved by the semi-analytical methods, between $\varphi=10^{-11}$ and $10^{-3}$. On the other hand, as for the shape design variable, the CVSA method showed stable accurate results (errors in the order of $10^{-12} \%$ ) for any perturbation value considered, including values as low as $\varphi=10^{-300}$ (minimum tested).

\section{CONCLUSIVE REMARKS}

- There is a considerable gain in storage and time requirements in both, the complex and real semi-analytical sensitivity methods when compared to the global counterparts. In the semianalytical cases, there is no need to define any global array as complex. All the complex computations are performed at the element level.

- Compared to global finite differences, the semi-analytical alternatives show higher time efficiency, since it is not necessary to solve a new global system of equations for each design variable nor to perform arithmetic operations with global complex matrices.

- The CVSA can easily outperform the RVSA in accuracy, provided that diminute perturbations are applied. In the examples studied, the CVSA method showed accuracy of results from moderate perturbation factors to values as low as $10^{-300}$. This contrasts with methods based on real variables, which show accuracy only for comparatively small perturbation ranges. 
- The presence of rigid rotations affects negatively the accuracy of sensitivity measures with respect to shape design variables, yet this drawback does not occur for the material design variables. This phenomenon is in accordance to the well-known pathology of the semi-analytical methods and shows up only for moderate to large perturbation factors.

- In the cellular beam-like problem, a sensitivity measure was monitored as the number of cells was increased. For a moderate perturbation factor $\left(\varphi=10^{-2}\right)$, linear and geometrically non-linear analyses presented different behaviors. In the linear case, the CVSA and CFD-RVSA methods presented similar growth pattern and error levels, one order lower than the FFD-RVSA approach but still unacceptable. In the nonlinear case, the CVSA method yielded approximately constant errors in the range of $2.5-3.5 \%$ for up to 60 cells. In opposition, the FFD-RVSA showed a steep error growth tending to a constant threshold as the number of cells was incremented. Noteworthy, as the number of cells increases, the problem becomes increasingly stretch-dominated and the number of elements subjected to rigid rotations becomes percentually smaller, reducing the influence of the associated pathology.

- In nonlinear analyses, the tolerance imposed to solve the nonlinear equilibrium equations plays a key role in the quality of results and should be made as tight as possible.

- The set of properties highlighted evince that the pathology associated to rigid rotations shows up irrespective if the analysis is linear or nonlinear in both, the RVSA and the CVSA. However, for sufficiently small perturbation factors this drawback is circumvented. As the CVSA can be used with no loss of accuracy for perturbation factors as low as $10^{-300}$, this method is prone to be employed as a black box for material or shape design variables. Notwithstanding, further tests involving elasticity, beam and plate elements subjected to rigid rotations are recommended.

\section{Acknowledgements}

The authors wish to express their gratitude to CNPq and CAPES (Brazilian research supporting agencies), and to UDESC for the concession of Master's scholarships associated to this work.

\section{References}

Barthelemy, B., Chon, C. T., Haftka, R. T., "Accuracy Problems Associated with Semi-Analytical Derivatives of Static Response", Finite Elements in Analysis and Design 4, pp 249-265, 1988.

Barthelemy, B., Haftka, R. T., "Accuracy Analysis of the Semi-Analytical Method for Shape Sensitivity Calculation", Mech. Struct. \& Mach, 18(3), pp 407-432, 1990.

Bletzinger, K., Firl, M., Daoud, F., "Approximation of Derivatives in Semi-Analytical Structural Optimization", Computers \& Structures 86, pp 1404-1416, 2007.

Boer, H. DE, Keulen, V. F., "Refined Semi-Analytical Design Sensitivities", Int. J. of Solids and Structures, 37, pp. 6961-6980, 2000.

Burg, C. O. E., Newman III, J. C., "Computationally Efficient, Numerically Exact Design Space Derivative Via the Complex Taylor's Series Expansion Method", Computers \& Fluids 32 (2003), pp. 373-383, 2001.

Cheng, G., Olhoff N., "Rigid Body Test Against Error in Semi-Analytical Sensitivity Analysis", Computers \& Structures Vol. 46, No.3, pp. 515-527, 1991.

Latin American Journal of Solids and Structures 12 (2015) 980-1005 
Choi, K. K., Kim, N. H., "Structural Sensitivity Analysis and Optimization 2: Nonlinear Systems and Applications", Mechanical engineering series, Springer, USA, 2005.

Crisfield, M. A., "Non-linear Finite Element Analysis of Solids and Structures", Vol. 1: Essentials, John Wiley \& Sons, 1991.

Hughes, T.J.R., Hilton, E., "Finite Element Methods for Plate and Shell Structures: Formulations and Algorithms", Vol. 2: Pineridge Press International, 1986.

Jin, W., "Semi-Analytical Complex Variable Based Stochastic Finite Element", PhD Thesis, University of Texas, 2008.

Jin, W., Dennis, B. H., Wang, B. P., "Improved Sensitivity and Reliability Analysis of Nonlinear Euler-Bernoulli Beam Using a Complex Variable Semi-Analytical Method", Proceedings of the ASME 2009 International Design Engineering Technical Conferences \& Computers and Information in Engineering Conference IDETC/CIE, 2009.

Jin, W., Dennis, B. H., Wang, B. P., "Improved Sensitivity Analysis Using a Complex Variable Semi-analytical Method", Structural and Multidisciplinary Optimization 41, pp. 433-439, 2010.

Kawamoto, A., "Path-Generation of Articulated Mechanisms by Shape and Topology Variations in Non-Linear truss Representation", Int. J. Num. Meth. Eng., pp 1557-1554, 2005.

Keulen, V. F., Haftka R. T., Kim N. H., "Review of Options for Structural Design Sensitivity Analysis. Part 1: Linear Systems", Comp. Meth. Appl. Mech. Eng. 194, pp. 3213-43, 2005.

Kleiber, M., Antúnez, H., Hien, T. D. E Kowalczyc, P., "Parameter Sensitivity in Nonlinear Mechanics: Theory and Finite Element Computations", John Wiley \& Sons Ltd., England, 1997.

Lyness, J. N., Moler, C. B., "Numerical Differentiation of Analytic Functions", SIAM J. Num. Anal., Vol. 4, pp. 2022010, 1967.

Mlejnek, H. P., "Accuracy of Semi-Analytical Sensitivities and its Improvement by the "Natural Method", Structural Optimization, pp.128-31, 1992.

Martins, J. R. R. A., Kroo, I. M., Alonso. J. J., "An Automated Method for Sensitivity Analysis Using Complex Variables", American Institute of Aeronautics and Astronautics, 2000.

Montoya, A., Fielder, R.., Gomez-Farias, A., Millwater, H., "Finite-Element Sensitivity for Plasticity Using Complex Variable Methods", Journal of Engineering Mechanics, Vol. 141(2), 2015.

Mundstock, D. C., "Shape Optimization using the Boundary Element Method and Sensitivity Calculation using Complex Variables". Master's Dissertation (in Portuguese), Universidade Federal do Rio Grande do Sul, 2006.

Mundstock, D.C., Marczak, R. J., "Boundary element sensitivity evaluation for elasticity problems using complex variable method", Structural and Multidisciplinary Optimization 38, pp. 423-428, 2009.

Newmann III, J. C., Anderson, W. K., Whitfield, D. L., "Multidisciplinary Sensitivity Derivatives Using Complex Variables", Computational Fluid Dynamics Laboratory NSF Engineering Research Center for Computational Field Simulation, MSSU-COE-ERC-98-08, 1998.

Olhoff, N., Rasmussen, J., Lund E., "A Method of "Exact" Numerical Differentiation for Error Elimination in FiniteElement-Based Semi-Analytical Shape Sensitivity Analyses", Mech. Struct. \& Mach., 21(1), pp. 1-66, 1993.

Parente, E., Vaz, L. E., "Improvement of Semi-Analytical Design Sensitivities of Non-Linear Structures Using Equilibrium Relations", Int. J. Num. Eng., vol. 50, pp 2127-2142, 2001.

Pedersen, P., Cheng, G., Rasmussen, J., "On Accuracy Problems for Semi-Analytical Sensitivity Analyses", Mech. Struct. \& Mach., 17(3), 373-384, 1989.

Stahlschmidt, J., "Sensitivity Analysis in Nonlinear Problems via the Semi-Analytical Method using Complex Variables: Application to Material and Geometric Design Variables", Master's Dissertation (in Portuguese), Universidade do Estado de Santa Catarina-UDESC, 2013. 